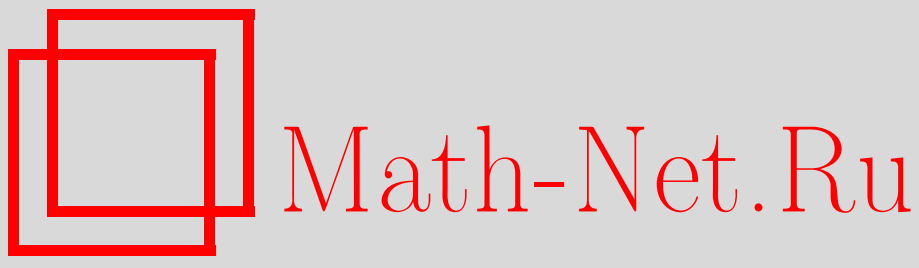

С. А. Назаров, Весовое анизотропное неравенство Корна для сочленения пластины со стержнями, Матем. сб., 2004, том 195, номер 4, 97-126

DOI: https://doi.org/10.4213/sm815

Использование Общероссийского математического портала Math-Net.Ru подразумевает, что вы прочитали и согласны с пользовательским соглашением

http://www . mathnet.ru/rus/agreement

Параметры загрузки:

IP : 44.207 .124 .84

26 апреля 2023 г., 13:20:52 
УДК 517.946

\author{
С. А. Назаров
}

\title{
Весовое анизотропное неравенство Корна для сочленения пластины со стержнями
}

В работе доказьвается неравенство Корна для упругого тела, полученного присоединением к пластине нескольких стержней с защемленными дальними торцами. Толщина пластины и диаметры стержней характеризуются общим малњтм параметром $h$, с которым соотносятся и различия в упругих свойствах элементов сочленения. Асимптотическая точность неравенства достигается подбором весовых и анизотропных норм, различающих продольные и поперечные направления в пластине и стержне, и подтверждается примерами конкретных конструкций. Как промежуточные получены новые результаты об одиночных пластинах и стержнях.

Библиографияя: 39 названий.

\section{§1. Постановка задачи и предварительное описание результатов}

1.1. Геометрия сочленений. Пусть $\omega^{0}, \omega^{1}, \ldots, \omega^{J}$ - области на плоскости $\mathbb{R}^{2}$, имеющие липшицевы границы и компактные замыкания. Обозначив через $h>0$ малый параметр, определим тонкую пластину

$$
\Omega_{h}^{0}=\left\{x=(y, z) \in \mathbb{R}^{3}: y=\left(y_{1}, y_{2}\right) \in \omega^{0}, \zeta:=h^{-1} z \in \Upsilon=\left(-\frac{1}{2}, \frac{1}{2}\right)\right\} .
$$

Пусть еще $Q_{h}^{1}, \ldots, Q_{h}^{J}$ - тонкие цилиндры с осями $\Lambda^{1}, \ldots, \Lambda^{J}$, пересекающими множество $\Sigma^{0}=\omega^{0} \times\{0\} \subset \mathbb{R}^{3}$ (срединную плоскость пластины),

$$
Q_{h}^{j}=\left\{x \in \mathbb{R}^{3}: \eta^{j}=\left(\eta_{1}^{j}, \eta_{2}^{j}\right):=h^{-1} y^{j} \in \omega^{j}, z^{j} \in \mathbb{R}\right\}
$$

здесь $x^{j}=\left(y^{j}, z^{j}\right)$ - декартовые координаты, причем $y^{j}=\left(y_{1}^{j}, y_{2}^{j}\right)$ - координаты в плоскостях, перпендикулярных $\Lambda^{j}$. Считаем, что сечение $\omega^{j}$ содержит начало координат $y^{j}=0$.

При описании стержней различаются два случая: стержень $G_{h}^{j}$ вставлен в паз $g_{h}^{j}=G_{h}^{j} \cap \Omega_{h}^{0}$ или приклеен к пластине вдоль поверхности $\mathscr{T}_{h}^{j} \subset \partial \Omega_{h}^{0}$. В первом случае $G_{h}^{j}$ отсекается от цилиндра (1.2) двумя плоскостями; торец, заглубленный в пластину, обозначаем $\Gamma_{h}^{j} \subset \Omega_{h}^{0}$, а другой $-\Gamma_{h}^{j 0}$. Под $\Gamma_{h}^{j}(t)=\left\{x \in Q_{h}^{j}: z^{j}=t\right\}$ подразумевается сечение цилиндра $Q_{h}^{j}$, параллельное торцу $\Gamma_{h}^{j}$ и проходящее через точку $z^{j}=t$ на оси $\Lambda^{j}$ (координату $z^{j}$ выбираем так, чтобы $\left.\Gamma_{h}^{j}=\Gamma_{h}^{j}(0)\right)$. Другими

Работа выполнена при финансовой поддержке Российского фонда фундаментальных исследований (грант № 00-01-00455). 
словами, стержень определяется формулой $G_{h}^{j}=\left\{x \in Q_{h}^{j}: z^{j} \in\left(0, l_{j}\right)\right\}$, где $l_{j}>0$ - длина стержня. Во втором случае стержень реально занимает объем $G_{h}^{j} \backslash \overline{\Omega_{h}^{0}}$ и отсекается от $Q_{h}^{j}$ поверхностью $\mathscr{T}_{h}^{j}=G_{h}^{j} \cap \partial \Omega_{h}^{0}$, однако для унификации обозначений такой стержень наращивается частью $g_{h}^{j}$ пластины и называется составным (см. далее п. 2.3).

Сочленение пластины и стержней - область $\Omega(h)$, замькание которой имеет вид

$$
\overline{\Omega(h)}=\overline{\Omega_{h}^{0}} \cup \overline{G_{h}^{1}} \cup \cdots \cup \overline{G_{h}^{J}} .
$$

Форма элементов сочленения (цилиндрические тела) упрощена сознательно и в $§ 4$ обсуждаются всевозможные обобщения. Кроме того, далее в п. 1.4 налагаются дополнительные ограничения на множества $g_{h}^{j}$.

Имея в виду проблему, сформулированную в [1; с. 293] более тридцати лет назад, называем пластину $\Omega_{h}^{0}$ столешней, а стержни $G_{h}^{j}-$ ножками вне зависимости от их положения относительно $\Omega_{h}^{0}$.

1.2. Неравенство Корна. Интерпретируя (1.3) как упругое тело, рассмотрим поле смещений $u=\left(u_{1}, u_{2}, u_{3}\right)^{\top}$, принадлежащее пространству Соболева $\stackrel{\circ}{H}^{1}\left(\Omega(h) ; \Gamma^{0}(h)\right)^{3}$; здесь “Т” - знак транспонирования, а символ “о " указывает на условия Дирихле

$$
u(x)=0, \quad x \in \Gamma^{0}(h)=\Gamma_{h}^{10} \cup \cdots \cup \Gamma_{h}^{J 0} .
$$

Механическая интерпретация условий (1.4) - жсесткое защемление торцов $\Gamma_{h}^{j 0}$, а принадлежность смещений упомянутому пространству интерпретируется как идеальный контакт стержней с пластиной.

Известно (см., например, [2]-[5]) неравенство Корна

$$
\left\|u ; H^{1}(\Omega(h))\right\|^{2} \leqslant c(h) \mathscr{E}(u ; \Omega(h))
$$

в котором $c(h)$ - постоянная, определяемая областью $\Omega(h)$, а $\mathscr{E}-$ функционал, подобный упругой энергии,

$$
\begin{aligned}
\mathscr{E}(u ; \Omega(h)) & =\sum_{i, k=1}^{3} \int_{\Omega(h)} \varepsilon_{i k}(u ; x)^{2} d x \\
\varepsilon_{i k} & =\frac{1}{2}\left(\frac{\partial u_{i}}{\partial x_{k}}+\frac{\partial u_{k}}{\partial x_{i}}\right) .
\end{aligned}
$$

Величины (1.7) представляют собой декартовы компоненты тензора деформаций.

Неравенство Корна играет центральную роль в математической теории упругости, однако из-за невыясненной зависимости константы Корна $c(h)$ от естественного малого параметра $h$ форма (1.5) записи этого неравенства становится бесполезной во многих практически важных вопросах. Целью статьи является доказательство весового анизотропного неравенства Корна

$$
\left\|u ; \Omega_{h}^{0}\right\|_{0}^{2}+\gamma(h) \sum_{j=1}^{J}\left\|u ; G_{h}^{j}\right\|_{j}^{2} \leqslant \mathscr{E}_{\gamma}(u ; \Omega(h))
$$


где $c$ - постоянная, не зависяшая ни от поля $u$, ни от параметра $h \in\left(0, h_{0}\right]$, а множитель $\gamma(h)$ отражает возможное различие упругих свойств пластины и стержней,

$$
\mathscr{E}_{\gamma}(u ; \Omega(h))=\left\{\begin{array}{l}
\mathscr{E}\left(u ; \Omega_{h}^{0} \backslash \bigcup_{j=1}^{J} G_{h}^{j}\right)+\gamma(h) \sum_{j=1}^{J} \mathscr{E}\left(u ; G_{h}^{j}\right) \\
\mathscr{E}\left(u ; \Omega_{h}^{0}\right)+\gamma(h) \sum_{j=1}^{J} \mathscr{E}\left(u ; G_{h}^{j} \backslash \Omega_{h}^{0}\right) .
\end{array}\right.
$$

Верхняя строчка (1.9) относится к случаю заглубленных стержней, а нижняя к случаю поверхностного сочленения. В $(1.8)$ и $(1.9)$ величина $\gamma(h)$ связывается c $h$, но в принщипе ее можно считать независимой - в окончательных формулах фигурируют выражения

$$
\lambda_{q}(h)=\min \left\{\gamma(h), h^{-q}|\log h|^{-2}\right\}
$$

с показателями $q \in\{0, \pm 1, \ldots\}$. Чтобы исключить равенство $\log h=0$, всюду далее считаем, что $h_{0}<1$ в ограничении $h \leqslant h_{0}$.

Асимптотическая точность неравенства Корна (1.8) достигается путем подбора специальных, зависящих от параметра $h$ норм $\|\cdot\|_{0}$ и $\|\cdot\|_{j}$ в пространствах $H^{1}\left(\Omega_{h}^{0}\right)^{3}$ и $\stackrel{\circ}{H^{1}}\left(G_{h}^{j} ; \Gamma_{h}^{j 0}\right)^{3}$.

1.3. Содержание статьи. В отличие от скалярных задач исследование сочленений сингулярно вырождающихся областей с различными предельными размерностями в теории упругости (стержней, пластин и обычных, не тонких, тел) подразумевает применение весовых и анизотропных соболевских норм, учитывающих различие эффективных свойств тонких упругих тел в продольных и поперечных направлениях, а также взаимное расположение элементов конструкций. Подобные нормы оказываются наиболее приспособленными к отслеживанию зависимости решений от малых параметров, а по достижении асимптотической точности сопутствующие неравенства Корна позволяют предугадывать асимптотические анзацы и максимально ослаблять требования к гладкости правых частей краевой задачи. Показательной в этом плане является ситуация с однородными сочленениями тело/стержни ("кактус"): при использовании изотропных норм в [6] и [7], [8] константа Корна была оценена сверху величинами $c h^{-10}$ и $c h^{-8}$ соответственно, в то время как правильной мажорантой, найденной на основе анизотропных норм и подтвержденной асимптотическим анализом, является $c h^{-4}$, причем для определенњых конфигураций она уменњшается до $\mathrm{ch}^{-2}$ (см. [9]-[11]).

Впервые анизотропное неравенство Корна возникло по-видимому в работе [12] при обосновании теории пластин (ср. лемму 2.1). Затем в [13] и [14] были установлены весовые и анизотропные неравенства Корна для иилиндрических пластин и стержней, а в [15], [16] и [14], [17] они были перенесены на случаи слабоизогнутых или локально периодических тонких тел с переменньм сечением (см. также [18; гл. 3]). Такие неравенства для элементов сочленения $\Omega_{h}^{0}$ и $G_{h}^{j}$ воспроизводятся в $\S 2$ и, кроме того, доказываются новые асимптотически точные неравенства Корна для составных стержней и пластин, защемленных по мальм множествам (см. п. 2.3 и $\S 4)$. 
Упомянем математические исследования упругих конструкций из тонких элементов, относящиеся как к выводу неравенств Корна, так и к собственно асимптотическому анализу. Наиболее полно изучены сочленения тело/пластина и тело/стержни (см. соответственно [19]-[21] и [6]-[11], [22]-[28], а также другие работы). Одномерным моделям стержневых сочленений и задачам осреднения решетчатых и рамных конструкций посвящены публикации [29]-[38] и др., однако до сих пор многие вопросы в этом направлении остаются открытыми. Какие-либо работы об упругих сочленениях пластина/стержни автору неизвестны (впрочем, скалярные уравнения на таких областях рассматривались в [39], [24]).

Неравенства Корна для области $\Omega(h)$ из (1.3) при разнообразных конфигурациях стержней установлены в $\S 3$. Так как торцы стержней зашемлены, адекватные нормы $\|\cdot\|_{j}$ известны (см. [14], [18] и лемму 2.3), а для образования подходящей нормы $\|\cdot\|_{0}$ используются прием отшепления жесткого смешения от сужения $\left.u\right|_{\Omega_{h}^{0}}$, разработанный в [10], и аккуратные оценки поля $u$ на пазах $g_{h}^{j}$, проверенные в $\S 2$. Отличительная черта сочленения пластина/стержни - рассогласование ослабленных (поперечных) направлений у элементов: у стержня таких направлений два, а у пластины - одно. Поэтому первостепенное значение приобретают направления осей стержней и положение малых зон соединений, причем приходится различать сочленения с одним, двумя, тремя и шестью ${ }^{1}$ стержнями, а в последних двух случаях возникают дополнительные геометрические ограничения. Подчеркнем, что при определенных требованиях к расположению стержней и к их жесткостям ("очень жесткие" (3.35) и “не очень мягкие" (3.45)) удается в качестве \|\|$_{0}$ взять соответственно нормы (3.34), (3.44) и (3.48), (3.49), которые лишш множителем $|\log h|^{-1}$ отличаются от нормы $|\cdot|_{0}$, характерной для защемленной пластины (см. [12], [13], [18] и формулу (2.4) в §2). Интересна механическая интерпретация этих результатов: для фиксации пластины достаточно двух (трех вертикальных) “очень жестких" ножек или шести “не очень мягких" растяжек. Кроме того, в 33 отыскиваются конфигурации стержней, гарантируюшие наилучшее закрепление пластины в поперечном или продольных направлениях. Приводятся примеры, подтверждающие асимптотическую точность установленных вариантов неравенства Корна и необходимость добавления логарифмических множителей в норму $\|\cdot\|_{0}$.

В последнем, четвертом, параграфе обсуждаются всевозможные обобщения и, в частности, случай мягких стержней, вставленных в пазы (он исключен из рассмотрения в $\S 3$ ).

1.4. О форме пазов. Как проверено в [18] для пластин и стержней, неравенства Корна и главные члены асимптотических разложений остаются в силе при достаточно произвольной форме кромки пластины и концов стержня. Разумеется, то же самое можно сказать и о поверхности, по которой стержень $G_{h}^{j}$ соединен с пластиной. Откажемся от упрощенных определений множеств $g_{h}^{j}$ и $\mathscr{T}_{h}^{j}$, данных ранее, и перечислим те их свойства, которые используются в следующих параграфах для вывода вспомогательных, но имеющих принципиальное значение неравенств.

Введем бесконечный круговой цилиндр $\mathbf{Q}_{h}^{j}=\left\{x:\left|\eta^{j}\right|<R_{j}\right\}$, содержащийся

\footnotetext{
${ }^{1}$ Конечно же, стержней может быть больше, но считаются только те, которые принимаются во внимание при выводе неравенства Корна.
} 
в цилиндре (1.2), а также его части

$$
\mathbf{g}_{h}^{j}=\left\{x \in \mathbf{Q}_{h}^{j}: z^{j} \in\left(h l_{-}^{j}, h l_{+}^{j}\right)\right\}, \quad \mathbf{g}_{h}^{j 0}=\left\{x \in \mathbf{Q}_{h}^{j}: z^{j} \in\left(h l_{-}^{j 0}, h l_{+}^{j 0}\right)\right\}
$$

при этом $R_{j}$ и $0 \leqslant l_{-}^{j}<l_{+}^{j} \leqslant l_{-}^{j 0}<l_{+}^{j 0}-$ размеры, не зависящие от $h$. Поверхность $\mathscr{T}_{h}^{j}$ отсекает от стержня $G_{h}^{j}$ малое множество $g_{h}^{j}$, а поверхность $\Gamma_{h}^{j}\left(h l_{+}^{j 0}\right)$ отсекает от остатка $G_{h}^{j} \backslash \bar{g}_{h}^{j}$ еще одно малое множество $g_{h}^{j 0}$. Радиус $R_{j}$ и длины $l_{ \pm}^{j}, l_{ \pm}^{j 0}$ выбраны так, что $\mathbf{g}_{h}^{j} \subset g_{h}^{j}, \mathbf{g}_{h}^{j 0} \subset g_{h}^{j 0}$ и поверхность раздела $\mathscr{T}_{h}^{j}$ не имеет общих точек с тонкими короткими цилиндрами $\mathbf{g}_{h}^{j}$ и $\mathbf{g}_{h}^{j 0}$.

Предполагаем, что

1) существуют два шара $B_{h}^{j} \subset \mathbf{g}_{h}^{j} u B_{h}^{j 0} \subset \mathbf{g}_{h}^{j 0}$ с радиусами $h R_{j}$, обеспечивающие следующие неравенства Корна:

$$
\left\|u ; g_{h}^{j \alpha}\right\|^{2} \leqslant c_{j}\left(\mathscr{E}\left(u ; g_{h}^{j \alpha}\right)+h^{-2}\left\|u ; L_{2}\left(B_{h}^{j \alpha}\right)\right\|^{2}\right)
$$

здесь $\alpha=0$ или $\alpha$ - пустое место, а норма $\|\cdot\|$ определена формулой

$$
\|u ; \Xi\|=\left(\left\|\nabla_{x} u ; L_{2}(\Xi)\right\|^{2}+h^{-2}\left\|u ; L_{2}(\Xi)\right\|^{2}\right)^{1 / 2} ;
$$

2) существуют куб $\mathscr{K}_{h}^{j} \supset g_{h}^{j}$ с ребром $\mathscr{A}_{j}$, шар $\mathscr{B}_{h}^{j} \subset \mathscr{K}_{h}^{j} \backslash g_{h}^{j}$ с радиусом $h \mathscr{R}_{j}$ и оператор продолжения $\beta^{j}: H^{1}\left(\mathscr{K}_{h}^{j} \backslash g_{h}^{j}\right) \rightarrow H^{1}\left(\mathscr{K}_{h}^{j}\right)$, для которьх

$$
\begin{gathered}
\left\|u ; \mathscr{K}_{h}^{j} \backslash g_{h}^{j}\right\|^{2} \leqslant c_{j}\left(\mathscr{E}\left(u ; \mathscr{K}_{h}^{j} \backslash g_{h}^{j}\right)+h^{-2}\left\|u ; L_{2}\left(\mathscr{B}_{h}^{j}\right)\right\|^{2}\right) \\
\left\|\beta^{j} u ; \mathscr{K}_{h}^{j}\right\| \leqslant C_{j}\left\|u ; \mathscr{K}_{h}^{j} \backslash g_{h}^{j}\right\| .
\end{gathered}
$$

Постоянные $c_{j}, C_{j}$ и размеры $l_{j}, R_{j} u \mathscr{A}_{j}, \mathscr{R}_{j}$ не зависят от параметpa $h$.

Пусть цилиндрический стержень присоединен к одному из оснований пластины и его ось $\Lambda^{j}$ пересекает множество $\Sigma^{0}$ в одной точке $P^{j}(h)$. Тогда при помощи растяжения в $h^{-1}$ раз координат относительно центра $P^{j}(h)$ области $g_{h}^{j}$ и $g_{h}^{j 0}$ переводятся в фиксированные (не зависящие от $h$ ) области, а значит, свойства 1) и 2 ) становятся очевидными. Если стержень прикреплен вблизи боковой поверхности пластины, то после растяжения координат области $g_{h}^{j}$ и $g_{h}^{j 0}$ не утрачивают зависимость от $h$. Тем неменее благодаря известным результатам (см. [5]) свойства 1) и 2) легко проверить и в этом случае (пояснения можно найти в $[18 ; \S \S 3.3,3.4]$ ). Итак, упомянутые требования становятся ограничительными лишь тогда, когда цилиндрическая форма стержня существенно изменена вблизи его конца, вставленного в паз.

Упомянем, наконец, бесспорное условие: при $h \rightarrow+0$ точка $P^{j}(h)$ стремится к фиксированной точке $P^{j}=P^{j}(0)$ и, кроме того, $\left|P^{j}(h)-P^{j}(0)\right| \leqslant c h$. Если ось $\Lambda^{j}$ лежит в плоскости $\{x: z=0\}$ и пересечение $\Lambda^{j} \cap \overline{\Sigma^{0}}-$ отрезок, то в качестве $P^{j}(h)=P^{j}$ берется точка на контуре $\partial \omega^{0} \times\{0\}$. 


\section{§2. Неравенства Корна для одиночных элементов}

2.1. Пластина. Введем матрицу

$$
d(y, z)=\left(\begin{array}{cccccc}
1 & 0 & 0 & 0 & z & -y_{2} \\
0 & 1 & 0 & -z & 0 & y_{1} \\
0 & 0 & 1 & y_{2} & -y_{1} & 0
\end{array}\right),
$$

порождаюшую жесткие смещения $d(x) a$, при этом $a$ - столбец из $\mathbb{R}^{6}$, а векторы $a^{\prime}=\left(a_{1}, a_{2}, a_{3}\right)^{\top}$ и $a^{\prime \prime}=\left(a_{4}, a_{5}, a_{6}\right)^{\top}$ ассоциируются с поступательными и вращательными смещениями.

Лемма 2.1. Для поля $v \in H^{1}\left(\Omega_{h}^{0}\right)^{3}$, подчиненного условию ортогональнос$m u$

$$
\int_{\Omega_{h}^{0}} d(y, z)^{\top} v(y, z) d y d z=0 \in \mathbb{R}^{6}
$$

справедливо неравенство Корна

$$
\left|v ; \Omega_{h}^{0}\right|_{0}^{2} \leqslant c \mathscr{E}\left(v ; \Omega_{h}^{0}\right)
$$

в котором постоянная с не зависит от и $и$ $h \in(0,1]$, а анизотропная норма $|\cdot|_{0}$ определена следующим образом:

$$
\begin{aligned}
\left|v ; \Omega_{h}^{0}\right|_{0}= & \left(\int _ { \Omega _ { h } ^ { 0 } } \left(\sum_{i=1}^{2}\left(\left|\frac{\partial v_{i}}{\partial y_{i}}\right|^{2}+h^{2}\left|\frac{\partial v_{i}}{\partial z}\right|^{2}+h^{2}\left|\frac{\partial v_{3}}{\partial y_{i}}\right|^{2}+\left|v_{i}\right|^{2}\right)\right.\right. \\
& \left.\left.+\left|\frac{\partial v_{3}}{\partial z}\right|^{2}+\left|\frac{\partial v_{1}}{\partial y_{2}}\right|^{2}+\left|\frac{\partial v_{2}}{\partial y_{1}}\right|^{2}+h^{2}\left|v_{3}\right|^{2}\right) d y d z\right)^{1 / 2} .
\end{aligned}
$$

ДокАЗАТЕльство неравенства (2.3), предложенное в [12] (см. также [18; теорема 3.2.1]), столь кратко, что здесь оно воспроизводится полностью. Делая замены

$$
z \mapsto \zeta=h^{-1} z, \quad v \mapsto V=\left(v_{1}, v_{2}, h v_{3}\right)^{\top},
$$

обнаруживаем, что при $h \leqslant 1$

$$
\begin{gathered}
\mathscr{E}\left(v ; \Omega_{h}^{0}\right)=\frac{h}{4} \int_{\Omega_{1}^{0}}\left(\sum_{i, k=1}^{2}\left|\frac{\partial V_{i}}{\partial y_{k}}+\frac{\partial V_{k}}{\partial y_{i}}\right|^{2}+2 h^{-2} \sum_{i=1}^{2}\left|\frac{\partial V_{i}}{\partial \zeta}+\frac{\partial V_{3}}{\partial y_{i}}\right|^{2}\right. \\
\left.+4 h^{-4}\left|\frac{\partial V_{3}}{\partial \zeta}\right|^{2}\right) d y d \zeta \geqslant h \mathscr{E}\left(V ; \Omega_{1}^{0}\right) \geqslant c h \int_{\Omega_{1}^{0}}\left(\sum_{i, k=1}^{2}\left|\frac{\partial V_{i}}{\partial y_{k}}\right|^{2}\right. \\
\left.+\sum_{i=1}^{2}\left(\left|\frac{\partial V_{i}}{\partial \zeta}\right|^{2}+\left|\frac{\partial V_{3}}{\partial y_{i}}\right|^{2}+\left|V_{i}\right|^{2}\right)+\left|\frac{\partial V_{3}}{\partial \zeta}\right|^{2}+\left|V_{3}\right|^{2}\right) d y d \zeta
\end{gathered}
$$

Последнее неравенство есть не что иное, как неравенство Корна в стандартной области $\Omega_{1}^{0}=\omega \times\left(-\frac{1}{2}, \frac{1}{2}\right)$, обеспеченное (см. [4; теорема 3.3 .3$]$ и [18; теорема 2.3.3]) условиями ортогональности

$$
\int_{\Omega_{1}^{0}} d(y, \zeta)^{\top} V(y, \zeta) d y d \zeta=0 \in \mathbb{R}^{6}
$$


которые вытекают из (2.2) и (2.5). Таким образом, постоянная $c>0$ в (2.6) не зависит от $h$, и остается, сделав обратные замены, уточнить, что возникающий множитель $h^{4}$ при $\left|\partial v_{3} / \partial z\right|^{2}$ устраним, поскольку деформация $\varepsilon_{33}(v)=\partial v_{3} / \partial z$ присутствует в самом функционале $\mathscr{E}\left(v ; \Omega_{h}^{0}\right)($ cp. $(1.7)$ с $(1.6))$.

ЗАмЕЧАнИЕ 2.2. Нетрудно убедиться в том, что $\varepsilon_{i k}(d a)=0$ и

$$
\mathscr{E}(v+d a ; \Xi)=\mathscr{E}(v ; \Xi) .
$$

2.2. Однородный стержень. В этом и следующем пунктах индекс $j$ у обозначений $G_{h}^{j}, \Gamma_{h}^{j},|\cdot|_{j}$ и др. не пишем, но помним, что координаты $y=y^{j}$ и $z=z^{j}$ привязаны к стержню, т.е. являются для него поперечными и продольной соответственно.

Очередная лемма, установленная в [14] (см. также [18; теорема 3.4.3]), обслуживает стержень, зашемленньй по торцу.

Лемма 2.3. Для поля $u \in \stackrel{\circ}{H^{1}}\left(G_{h} ; \Gamma_{h}^{0}\right)^{3}$ выполняется неравенство Корна

$$
\left|u ; G_{h}\right|^{2} \leqslant c \mathscr{E}\left(u ; G_{h}\right)
$$

в котором постоянная с не зависит от $u$ u $h$, а в весовой анизотропной норме

$$
\begin{aligned}
\left|u ; G_{h}\right|= & \left(\int _ { G _ { h } } \left(\sum_{i=1}^{2}\left(\left|\frac{\partial u_{i}}{\partial y_{i}}\right|^{2}+h^{2} \rho_{h}^{-2}\left(\left|\frac{\partial u_{i}}{\partial z}\right|^{2}+\left|\frac{\partial u_{3}}{\partial y_{i}}\right|^{2}\right)+h^{2} \rho_{h}^{-4}\left|u_{i}\right|^{2}\right)\right.\right. \\
& \left.\left.+\left|\frac{\partial u_{3}}{\partial z}\right|^{2}+h^{2} \rho_{h}^{-2}\left(\left|\frac{\partial u_{1}}{\partial y_{2}}\right|^{2}+\left|\frac{\partial u_{2}}{\partial y_{1}}\right|^{2}\right)+\rho_{h}^{-2}\left|u_{3}\right|^{2}\right) d y d z\right)^{1 / 2}
\end{aligned}
$$

присутствует множстель $\rho_{h}(y, z)=h+\operatorname{dist}\left\{(y, z), \Gamma_{h}^{0}\right\}$, равный $O(h)$ вблизи защемленного торца и $O(1)$ на удалении от него.

В следующей лемме содержатся оценки сужений поля $u$ на малые множества торец $\Gamma_{h}$ и концевую часть $g_{h}-$ и фигурируют средние по сечениям $\Gamma_{h}(t)$,

$$
\bar{u}_{i}(t)=\left(\operatorname{mes}_{2} \Gamma_{h}(t)\right)^{-1} \int_{\Gamma_{h}(t)} u_{i}(y, z) d s,
$$

компонент $u_{i}$, продолженных нулем за торец $\Gamma_{h}^{0}$ на полуцилиндр $Q_{h}^{+}($см. $(1.2))$.

Лемма 2.4. Пусть $и \in \stackrel{\circ}{H^{1}}\left(G_{h} ; \Gamma_{h}\right)^{3}$. Справедливо неравенство

$$
\sum_{i=1}^{2}\left(h^{2}\left\|u_{i} ; L_{2}\left(\Gamma_{h}(t)\right)\right\|^{2}+\left\|u_{i}-\bar{u}_{i} ; L_{2}\left(\Gamma_{h}(t)\right)\right\|^{2}\right)+\left\|u_{3} ; L_{2}\left(\Gamma_{h}(t)\right)\right\|^{2} \leqslant c \mathscr{E}\left(u ; G_{h}\right)
$$

в котором постоянная с не зависит от параметра $h \in(0,1]$, координать $t$ сечения $\Gamma_{h}(t)$ и поля $и$. Кроме того,

$$
\sum_{i=1}^{2}\left(h^{2}\left\|u_{i} ; L_{2}\left(g_{h}\right)\right\|^{2}+\left\|u_{i}-\bar{u}_{i} ; L_{2}\left(g_{h}\right)\right\|^{2}\right)+\left\|u_{3} ; L_{2}\left(g_{h}\right)\right\|^{2} \leqslant \operatorname{clh} \mathscr{E}\left(u ; G_{h}\right),
$$

әде с - та же постоянная, что и в (2.11), а длина $l$ выбрана так, чтобъ множество $g_{h}$ находилось мехду сечениями $\Gamma_{h}(0) u \Gamma_{h}(h l)$. 
ДокАЗАТЕЛЬСтво. Соотношение (2.12) возникает в результате интегрирования $(2.11)$ по $t \in(0, h l)$. В (2.11) оценки норм функций $u_{i}$ и $u_{3}$ вытекают из неравенства Корна (2.8), структуры весовой нормы (2.9) (отметим, что $\rho_{h} \leqslant \rho^{0}$ на $G_{h}$ ) и очевидного “следового" неравенства

$$
|v(T)|^{2} \leqslant \int_{T}^{+\infty}\left|\frac{d v}{d \tau}(\tau)\right|^{2} d \tau+2 \int_{T}^{+\infty}|v(\tau)|^{2} d \tau,
$$

которое опять-таки нужно проинтегрировать (теперь по сечению, взяв $T$ переменным). Наконец, оценки норм функций $u_{i}-\bar{u}_{i}$ обеспечиваются той же формулой (2.13), если установлено соотношение

$$
\left\|u_{i}-\bar{u}_{i} ; L_{2}\left(G_{h}\right)\right\|^{2}+\left\|\frac{\partial}{\partial z}\left(u_{i}-\bar{u}_{i}\right) ; L_{2}\left(G_{h}\right)\right\|^{2} \leqslant c \mathscr{E}\left(u ; G_{h}\right) .
$$

Применяя неравенство Пуанкаре на $\Gamma_{h}(z)$ и неравенство Корна $(2.8)$, видим, что

$$
\left\|u_{i}-\bar{u}_{i} ; L_{2}\left(G_{h}\right)\right\|^{2} \leqslant c h^{2}\left\|\nabla_{(y, z)} u_{i} ; L_{2}\left(G_{h}\right)\right\|^{2} \leqslant c\left|u_{i} ; G_{h}\right|^{2} \leqslant c \mathscr{E}\left(u ; G_{h}\right) .
$$

Подчеркнем, что множитель $h^{2}$, появившийся благодаря малости сечения $\Gamma_{h}(z)$, компенсирует множители $h^{2}$, имеюшиеся в (2.9). Для проверки оценки нормы производной в (2.14) разрежем стержень $G_{h}$ сечениями $\Gamma_{h}(p h l)$ на одинаковые куски $\Xi_{h}^{p}, p=1, \ldots, P_{h}$ (число $P_{h}$ выбираем так, чтобы объединение замыканий кусков накрывало $\left.G_{h}\right)$. На каждом из множеств $\Xi_{h}^{p}$ представим поле $u$ в виде ${ }^{2}$

$$
u(y, z)=v^{p}(y, z)+d(y, z-h l) a^{p},
$$

причем столбец $a^{p} \in \mathbb{R}^{6}$ подберем так, чтобы выполнялось условие ортогональности

$$
\int_{\Xi_{h}^{p}} d(y, z-h l)^{\top} v^{p}(y, z) d y d z=0 \in \mathbb{R}^{6}
$$

(ср. с (2.2)). Это условие обеспечивает неравенство Корна

$$
\left\|v^{p} ; L_{2}\left(\Xi_{h}^{p}\right)\right\|^{2} \leqslant c \mathscr{E}\left(v^{p} ; \Xi_{h}^{p}\right)=c \mathscr{E}\left(v^{p} ; \Xi_{h}^{p}\right),
$$

где $\|\cdot\|$ - норма (1.11) и постоянная $c$ не зависит от $u$ и $h, p$ (см. [18; п. 3.3.2], а также замечание 2.2$)$. Поскольку согласно (2.1) и (2.10)

$$
d(y, z-h l)-\bar{d}(z-h l)=\left(\begin{array}{cccccc}
0 & 0 & 0 & 0 & \delta_{3} & -y_{2}+\delta_{2} \\
0 & 0 & 0 & -\delta_{3} & 0 & y_{1}-\delta_{1} \\
0 & 0 & 0 & y_{2}-\delta_{2} & -y_{1}+\delta_{1} & 0
\end{array}\right),
$$

где $\delta_{p}$ - некоторые постоянные, имеем

$$
\frac{\partial}{\partial z}(u-\bar{u})=\frac{\partial}{\partial z}\left(v^{p}-\bar{v}^{p}\right)+\frac{\partial}{\partial z}(d-\bar{d}) a^{p}=\frac{\partial}{\partial z}\left(v^{p}-\bar{v}^{p}\right),
$$

\footnotetext{
${ }^{2}$ Выбор начала координат здесь несущественен, и обозначения приспосабливаются к доказательству леммы 2.5 в п. 2.3. По поводу определения столбца $a^{p}$ см. далее комментарии к фороруле (3.3).
} 
а значит, в силу (2.18)

$$
\begin{aligned}
& \left\|\frac{\partial}{\partial z}(u-\bar{u}) ; L_{2}\left(\Xi_{h}^{p}\right)\right\|^{2}=\left\|\frac{\partial}{\partial z}\left(v^{p}-\bar{v}^{p}\right) ; L_{2}\left(\Xi_{h}^{p}\right)\right\|^{2} \\
& \quad \leqslant 2\left\|\frac{\partial}{\partial z} v^{p} ; L_{2}\left(\Xi_{h}^{p}\right)\right\|^{2}+2\left\|\nabla_{(y, z)} v^{p} ; L_{2}\left(\Xi_{h}^{p}\right)\right\|^{2} \leqslant 4 c \mathscr{E}\left(u ; \Xi_{h}^{p}\right) .
\end{aligned}
$$

Просуммировав по $p=1, \ldots, P_{h}$ неравенства (2.19) (без их средних частей), приходим к оценке, которая вместе с (2.15) дает искомое соотношение (2.14).

2.3. Составной стержень. Если стержень $G_{h}^{j}$ заделывается в паз, то по лемме 2.3 в неравенстве (1.8) фигурирует норма $\left\|u ; G_{h}^{j}\right\|_{j}=\left|u ; G_{h}^{j}\right|_{j}$ из (2.9). Следующее утверждение предоставляет норму $\|\cdot\|_{j}$ в случае составного стержня.

ЛЕмма 2.5. Для поля $u \in \stackrel{\circ}{H^{1}}\left(G_{h}, \Gamma_{h}\right)^{3}$ выполняется неравенство

$$
\gamma(h)\left\|u ; G_{h}\right\|^{2} \leqslant c\left\{\mathscr{E}\left(u ; g_{h}\right)+\gamma(h) \mathscr{E}\left(u ; G_{h} \backslash g_{h}\right)\right\},
$$

в котором $c-$ не зависящая от $и$ и $h \in(0,1]$ постоянная $и$

$$
\begin{gathered}
\left\|u ; G_{h}\right\|=\left(\left|u ; G_{h} \backslash g_{h}\right|^{2}+\gamma(h)^{-1}\left\|u ; g_{h}\right\|^{2}\right)^{1 / 2} \\
\left\|u ; g_{h}\right\|=\left(\int _ { g _ { h } } \left(\sum_{i=1}^{2}\left(\left|\frac{\partial u_{i}}{\partial y_{i}}\right|^{2}+\mu_{2}(h) h^{1}\left(\left|\frac{\partial u_{i}}{\partial z}\right|^{2}+\left|\frac{\partial u_{3}}{\partial y_{i}}\right|^{2}\right)+\mu_{3}(h) h^{1}\left|u_{i}\right|^{2}\right)\right.\right. \\
\left.\left.+\left|\frac{\partial u_{3}}{\partial z}\right|^{2}+\mu_{1}(h) h^{1}\left(\left|\frac{\partial u_{1}}{\partial y_{2}}\right|^{2}+\left|\frac{\partial u_{2}}{\partial y_{1}}\right|^{2}\right)+\mu_{1}(h) h^{-1}\left|u_{3}\right|^{2}\right) d y d z\right)^{1 / 2}, \\
\mu_{q}(h)=\min \left\{\gamma(h), h^{-q}\right\} .
\end{gathered}
$$

ДокАЗАТЕльство. Сначала рассмотрим упрощенный случай $\mathscr{T}_{h}=\Gamma_{h}(h l)$ (т.е. $g_{h}=\Xi_{h}^{1}$ в обозначениях из п. 2.2 ), а затем, опираясь на предположение 1 ), распространим результат на пазы произвольной формы.

Трансформируем декартову систему так, чтобы ось $z$ прошла через центр тяжести фигуры $\Gamma_{h}(t)$, а проекции осей $y_{1}$ и $y_{2}$ на $\Gamma_{h}(t)$ совпали с главными осями инерции. Неравенства (2.8) и (2.11) остаются верньми и для укороченного стержня $G_{h} \backslash \bar{g}_{h}$ (так как поле $u$ продолжается нулем за сечение $\Gamma_{h}^{0}$, нарашиваем стержень в ту же сторону и делаем его длину не зависящей от $h)$. Используем представление $(2.16)$ на множестве $\Xi_{h}^{1}=g_{h}$. Умножив $(2.16)$ на $(6 \times 3)$-матрицу $d(y, z-h l)^{\top}$, проинтегрируем по $\Gamma_{h}(h l)$ и получим систему уравнений

$$
M a^{1}=F=\int_{\Gamma_{h}} d(y, 0)^{\top}\left(u(y, h l)-v^{1}(y, h l)\right) d s
$$

с диагональной $(6 \times 6)$-матрицей

$$
M=\int_{\Gamma_{h}} d(y, 0)^{\top} d(y, 0) d y=\operatorname{diag}\left\{h^{2} \sigma_{0}, h^{2} \sigma_{0}, h^{2} \sigma_{0}, h^{2} \sigma_{2}, h^{4} \sigma_{1}, h^{4}\left(\sigma_{1}+\sigma_{2}\right)\right\},
$$


где $h^{2} \sigma_{0}$ - площадь, а $h^{4} \sigma_{1}$ и $h^{4} \sigma_{2}$ - величины, пропорциональные главным моментам инерции фигуры $\Gamma_{h}$. Подчеркнем, что благодаря выбору системы координат интегралы от $y_{1}, y_{2}$ и $y_{1} y_{2}$ по $\Gamma_{h}$ равны нулю, а величины $\sigma_{k}>0$ не зависят от $h$.

Исходя из формул $(2.24)$ и $(2.25)$, оценим компоненты $a_{q}^{1}$ столбца $a^{1} \in \mathbb{R}^{6}$. Учитывая следовые неравенства (2.11) и

$$
h^{-1}\left\|v^{1} ; L_{2}\left(\Gamma_{h}(h l)\right)\right\|^{2} \leqslant c\left(h^{-2}\left\|v^{1} ; L_{2}\left(g_{h}\right)\right\|^{2}+\left\|\nabla_{(y, z)} v^{1} ; L_{2}\left(g_{h}\right)\right\|^{2}\right) \leqslant c \mathscr{E}\left(u ; g_{h}\right)
$$

(последнее обеспечено соотношением (2.18)), имеем

$$
\begin{aligned}
\left|a_{i}^{1}\right|^{2} & \leqslant c h^{-4}\left|F_{i}\right|^{2} \leqslant c h^{-4} \operatorname{mes}_{2} \Gamma_{h} \int_{\Gamma_{h}(h l)}\left(\left|v_{i}^{1}\right|^{2}+\left|u_{i}\right|^{2}\right) d s \\
& \leqslant c h^{-2}\left(h \mathscr{E}\left(u ; g_{h}\right)+h^{-2} \mathscr{E}\left(u ; G_{h} \backslash g_{h}\right)\right) \leqslant c h^{-4} \mu_{3}(h)^{-1} \mathscr{E}_{\gamma}\left(u ; G_{h}\right), \\
\left|a_{3}^{1}\right|^{2} & \leqslant c h^{-4}\left|F_{3}\right|^{2} \leqslant c h^{-2}\left(h \mathscr{E}\left(u ; g_{h}\right)+\mathscr{E}\left(u ; G_{h} \backslash g_{h}\right)\right) \\
& \leqslant c h^{-2} \mu_{1}(h)^{-1} \mathscr{E}_{\gamma}\left(u ; G_{h}\right), \\
\left|a_{6-i}^{1}\right|^{2} & \leqslant c h^{-8}\left|F_{6-i}\right|^{2} \leqslant c h^{-8} \max _{\Gamma_{h}}|d(y, 0)|^{2} \operatorname{mes}_{2} \Gamma_{h} \int_{\Gamma_{h}(h l)}\left(\left|v_{3}^{1}\right|^{2}+\left|u_{3}\right|^{2}\right) d s \\
& \leqslant c h^{-4} \mu_{1}(h)^{-1} \mathscr{E}_{\gamma}\left(u ; G_{h}\right), \\
\left|a_{6}^{1}\right|^{2} & \leqslant c h^{-8}\left|F_{6}\right|^{2} \leqslant c h^{-4} \int_{\Gamma_{h}(h l)}\left(\left|v_{1}^{1}\right|^{2}+\left|v_{2}^{1}\right|^{2}+\left|u_{1}-\bar{u}_{1}\right|^{2}+\left|u_{2}-\bar{u}_{2}\right|^{2}\right) d s \\
& \leqslant c h^{-4} \mu_{1}(h)^{-1} \mathscr{E}_{\gamma}\left(u ; G_{h}\right),
\end{aligned}
$$

здесь $i=1,2, \mathscr{E}_{\gamma}\left(u ; G_{h}\right)$ - сумма из фигурных скобок в $(2.20)$, а $\mu_{q}(h)$ - величина $(2.23)$.

Теперь для жесткого смешения $w^{1}(y, z)=d(y, z-h l) a^{1}$ имеем

$$
\begin{gathered}
\left\|w^{1} ; g_{h}\right\|^{2} \leqslant \\
c \operatorname{mes}_{2} g_{h}\left(\mu_{1}(h) h\left|\left(a^{1}\right)^{\prime \prime}\right|^{2}+\mu_{3}(h) h\left(\left|a_{1}^{1}\right|^{2}+\left|a_{2}^{1}\right|^{2}+h^{2}\left|\left(a^{1}\right)^{\prime \prime}\right|^{2}\right)\right. \\
\left.+\mu_{1}(h) h^{-1}\left(\left|a_{3}^{1}\right|^{2}+h^{2}\left|\left(a^{1}\right)^{\prime \prime}\right|^{2}\right)\right) \leqslant \mathscr{c}_{\gamma}\left(u ; G_{h}\right)
\end{gathered}
$$

(воспользовались тем, что $h^{2} \mu_{3}(h) \mu_{1}(h)^{-1} \leqslant 1$ ). Кроме того, сравнивая нормы (1.12) и (2.22), получаем в силу (2.18), что $\left\|v^{1} ; g_{h}\right\|^{2} \leqslant c\left\|v^{1} ; g_{h}\right\|^{2} \leqslant c \mathscr{E}\left(u ; g_{h}\right)$, а значит, в рассматриваемом упрощенном случае неравенство $(2.20)$ доказано.

В общей ситуации образуем более узкий стержень $\mathbf{G}_{h}$, отсекая его от кругового цилиндра $\mathbf{Q}_{h}$ поверхностями $\left\{x: z^{j}=h l_{-}^{j}\right\}$ и $\Gamma_{h}^{0}$ (см. п. 1.4). В качестве фиктивной разделяющей поверхности возьмем сечение $\mathbf{T}_{h}^{0}=\left\{x \in \mathbf{Q}_{h}: z^{j}=h l_{+}^{j}\right\}$, если $\gamma(h) \leqslant 1$, и $\mathbf{T}_{h}^{0}=\left\{x \in \mathbf{Q}_{h}: z^{j}=h l_{-}^{0}\right\}$ в противном случае. Такой выбор $\mathbf{T}_{h}^{0}$ обеспечивает соотношение

$$
\mathscr{E}\left(u ; \mathbf{g}_{h}\right)+\gamma(h) \mathscr{E}\left(u ; \mathbf{G}_{h} \backslash \mathbf{g}_{h}\right)=\mathscr{E}_{\gamma}\left(u ; \mathbf{G}_{h}\right) \leqslant \mathscr{E}_{\gamma}\left(u ; G_{h}\right)
$$

и поэтому согласно доказанному ранее справедливо неравенство (2.20) на круговом стержне $\mathbf{G}_{h}$. Далее понадобится еще одно неравенство

$$
h^{-1} \mu_{1}(h)\left\|u-\bar{u} ; L_{2}\left(B_{h}\right)\right\|^{2} \leqslant c \mathscr{E}_{\gamma}\left(u ; \mathbf{G}_{h}\right) \leqslant c \mathscr{E}_{\gamma}\left(u ; G_{h}\right),
$$


где теперь под $\bar{u}$ понимается среднее $u$ по шару $B_{h}$,

$$
\bar{u}=\left(\operatorname{mes}_{3} B_{h}\right)^{-1} \int_{B_{h}} u(x) d x .
$$

Поступательное смещение исчезает из разности $w^{1}(x)-\bar{w}^{1}(x)$, а значит, эта разность составляет $O(h)$ на $B_{h}$ и в силу $(2.29),(2.30)$

$$
h^{-1} \mu_{1}(h)\left\|w^{1}-\bar{w}^{1} ; L_{2}\left(B_{h}\right)\right\|^{2} \leqslant c h^{-1} \mu_{1}(h) h^{3} h^{2}\left|\left(a^{1}\right)^{\prime \prime}\right|^{2} \leqslant c \mathscr{E}_{\gamma}\left(u ; G_{h}\right) .
$$

Благодаря очевидному соотношению

$$
\begin{aligned}
h^{-1} \mu_{1}(h)\left\|v^{1}-\bar{v}^{1} ; L_{2}\left(B_{h}\right)\right\|^{2} & \leqslant h^{-2}\left\|v^{1}-\bar{v}^{1} ; L_{2}\left(B_{h}\right)\right\|^{2} \leqslant 2 h^{-2}\left\|v^{1} ; L_{2}\left(B_{h}\right)\right\|^{2} \\
& \leqslant 2\left\|v^{1} ; B_{h}\right\|^{2} \leqslant c \mathscr{E}\left(u ; g_{h}\right)
\end{aligned}
$$

(см. (2.18)) неравенство (2.31) установлено.

На шаре $B_{h} \subset \mathbf{g}_{h}$ с центром $\mathbf{P}(h)$ представим поле $u$ в виде

$$
u(x)=v(x)+d(x-\mathbf{P}(h)) a,
$$

где компонента $v$ подчиняется условию ортогональности

$$
\int_{B_{h}} d(x-\mathbf{P}(h))^{\top} v(x) d x=0 .
$$

При этом в силу неравенств Корна (2.18) и (1.11)

$$
\begin{aligned}
\left\|v ; g_{h}\right\|^{2} & \leqslant c\left(\mathscr{E}\left(v ; g_{h}\right)+h^{-2}\left\|v ; L_{2}\left(B_{h}\right)\right\|^{2}\right) \leqslant c\left(\mathscr{E}\left(v ; g_{h}\right)+\mathscr{E}\left(v ; B_{h}\right)\right) \\
& \leqslant 2 c \mathscr{E}\left(v ; g_{h}\right)=2 c \mathscr{E}\left(u ; g_{h}\right) .
\end{aligned}
$$

В п. 3.2 будут подробно разъяснены формулы

$$
\begin{gathered}
\left|a^{\prime}\right| \leqslant c h^{-3}\left|\mathbf{F}^{\prime}\right|, \quad\left|a^{\prime \prime}\right| \leqslant c h^{-5}\left|\mathbf{F}^{\prime \prime}\right|, \\
\mathbf{F}=\int_{B_{h}} d(x-\mathbf{P}(h))^{\top}(u(x)-v(x)) d x
\end{gathered}
$$

(ср. (3.12)-(3.14) и (3.15)). Следующие оценки, вполне аналогичные (2.27)-(2.30), опираются на неравенства (2.20) для $\mathbf{G}_{h}$ и (2.31), (2.35):

$$
\begin{aligned}
\left|a_{i}\right|^{2} & \leqslant c h^{-6}\left|\mathbf{F}_{i}\right|^{2} \leqslant c h^{-6} \operatorname{mes}_{3} B_{h} \int_{B_{h}}\left(\left|u_{i}\right|^{2}+|v|^{2}\right) d x \\
& \leqslant c h^{-3}\left(h^{-1} \mu_{3}(h)^{-1}\left\|u ; g_{h}\right\|^{2}+h^{2}\left\|v ; g_{h}\right\|^{2}\right) \leqslant c h^{-4} \mu_{3}(h)^{-1} \mathscr{E}_{\gamma}\left(u ; G_{h}\right), \quad\left(2.3{ }^{2}\right. \\
\left|a_{3}\right|^{2} & \leqslant c h^{-6}\left|\mathbf{F}_{3}\right|^{2} \leqslant c h^{-3}\left(h \mu_{1}(h)^{-1}\left\|u ; g_{h}\right\|^{2}+h^{2}\left\|v ; g_{h}\right\|^{2}\right) \\
& \leqslant c h^{-2} \mu_{1}(h)^{-1} \mathscr{E}_{\gamma}\left(u ; G_{h}\right), \\
\left|a^{\prime \prime}\right|^{2} & \leqslant c h^{-10}\left|\mathbf{F}^{\prime \prime}\right|^{2} \leqslant c h^{-10} \sup _{B_{h}}|d(x-\mathbf{P}(h))| \operatorname{mes}_{3} B_{h} \int_{B_{h}}\left(|u-\bar{u}|^{2}+|v|^{2}\right) d x \\
& \leqslant c h^{-5}\left(h \mu_{1}(h)^{-1} \mathscr{E}_{\gamma}\left(u ; G_{h}\right)+h^{2}\left\|v ; B_{h}\right\|^{2}\right) \leqslant c h^{-4} \mu_{1}(h)^{-1} \mathscr{E}_{\gamma}\left(u ; G_{h}\right) .
\end{aligned}
$$


Прямые вычисления нормы (2.22) жесткого смешения $w(x)=d(x-\mathbf{P}(h)) a$ показывают, что

$$
\begin{aligned}
\left\|w ; g_{h}\right\|^{2} & \leqslant c\left(h^{4} \mu_{3}(h)\left(\left|a_{1}\right|^{2}+\left|a_{2}\right|^{2}+h^{2}\left|a^{\prime \prime}\right|^{2}\right)+h^{2} \mu_{1}(h)\left(\left|a_{3}\right|^{2}+h^{2}\left|a^{\prime \prime}\right|^{2}\right)\right) \\
& \leqslant c\left(1+h^{2} \mu_{3}(h) \mu_{1}(h)^{-1}\right) \mathscr{E}_{\gamma}\left(u ; G_{h}\right) \leqslant 2 c \mathscr{E}_{\gamma}\left(u ; G_{h}\right) .
\end{aligned}
$$

Отсюда и из соотношения $\left\|v ; g_{h}\right\| \leqslant\left\|v ; g_{h}\right\|$, обеспеченного формулами (2.35) и (2.22), (1.12), вытекает первая из требуемых оценок

$$
\left\|u ; g_{h}\right\|^{2} \leqslant c \mathscr{E}_{\gamma}\left(u ; G_{h}\right) .
$$

Вторая оценка

$$
\left|u ; G_{h} \backslash g_{h}\right|^{2} \leqslant c \mathscr{E}\left(u ; G_{h} \backslash g_{h}\right)
$$

проверяется по той же схеме, что и первая, но шар $B_{h}^{1}$ заменяется шаром $B_{h}^{10}$, на котором, в частности, используются неравенства из леммы 2.4 и предположение 1 ) из п. 1.4.

ЗАмечАние 2.6. 1) Асимптотическая точность неравенства Корна (2.8) и правильность распределения весовых множителей в норме (2.9) подтверждены в [18; $\S 3.4]$ при помощи асимптотического анзаца, стандартного в теории тонких стержней. Этот же анзацподходит для проверки аналогичных свойств неравенства (2.20) и нормы (2.22).

2) Неравенство (2.31) прошло незамеченным, но оно сыграет немаловажную роль в $\S 3$.

2.4. Вспомогательные неравенства для пластины. Неравенство Корна (2.3) и одномерное неравенство Харди

$$
\int_{0}^{R}|V(r)|^{2}(1+|\log r|)^{-2} r^{-1} d r \leqslant c_{R}\left(\int_{0}^{R}\left|\frac{\partial V}{\partial r}(r)\right|^{2} r d r+\int_{R / 2}^{R}|V(r)|^{2} r d r\right),
$$

проинтегрированное по угловой, а также по поперечной переменным, доставляют следуюшее утверждение.

ЛЕмма 2.7. Для поля $v \in H^{1}\left(\Omega_{h}^{0}\right)^{3}$ справедливо соотношение

$$
\begin{aligned}
& h^{-1} \sum_{i=1}^{2}\left(\left\|v_{i} ; L_{2}\left(g_{h}^{j}\right)\right\|+h^{1 / 2}\left\|v_{i} ; L_{2}\left(\mathscr{T}_{h}^{j}\right)\right\|\right)+\left\|v_{3} ; L_{2}\left(g_{h}^{j}\right)\right\|+h^{1 / 2}\left\|v_{3} ; L_{2}\left(\mathscr{T}_{h}^{j}\right)\right\| \\
& \quad \leqslant c|\log h|\left|v ; \Omega_{h}^{0}\right|_{0},
\end{aligned}
$$

в котором постоянная с не зависит от $v$ и $h \in\left(0, h_{0}\right]$.

Напоминаем про соглашение: $h_{0}<1$, поэтому $\log h<0$.

Далее будет востребовано подобное (2.31) неравенство для разности $u_{3}-\bar{u}_{3}$, где $\bar{u}_{3}$ - среднее (2.32) функции $u_{3}$ по шару $B_{h}^{j}$.

ЛЕмма 2.8. Для $u \in H^{1}\left(\Omega_{h}^{0}\right)^{3}$ справедливо соотношение

$$
h^{-2}\left\|u_{3}-\bar{u}_{3} ; L_{2}\left(B_{h}^{j}\right)\right\|^{2} \leqslant c\left(|\log h|^{2}\left|u ; \Omega_{h}^{0}\right|^{2}+\mathscr{E}\left(u ; \Omega_{h}^{0}\right)\right),
$$

причем постоянная с не зависит от и $и ~ h \in\left(0, h_{0}\right]$. 
ДоКАЗАТЕЛЬСТво. Представим поле $u$ в виде (2.33) и заметим, что в силу (2.34) нужная оценка для слагаемого $v_{3}$ содержится в (2.35). Рассмотрим жесткое смещение $w(x)=d\left(x-\mathbf{P}^{j}(h)\right) a$. Благодаря (2.1) выполняются соотношения

$$
\begin{gathered}
w_{3}(x)-\bar{w}_{3}=a_{4}\left(y_{2}-\mathbf{P}_{2}^{j}(h)\right)-a_{5}\left(y_{1}-\mathbf{P}_{1}^{j}(h)\right), \\
h^{-2}\left\|w_{3}-\bar{w}_{3} ; L_{2}\left(B_{h}^{j}\right)\right\|^{2} \leqslant c h^{-2} h^{3} h^{2}\left(\left|a_{4}\right|^{2}+\left|a_{5}\right|^{2}\right) .
\end{gathered}
$$

Кроме того, согласно той же формуле (2.1) для матрицы $d(x)$ имеем

$$
\left|a_{6-i}\right|=\left[\frac{4}{15} \pi R_{j}^{5} h^{5}\right]^{-1}\left|\int_{B_{h}^{j}}\left(z-\mathbf{P}_{3}^{j}(h)\right) w_{i}(x) d x\right|
$$

(в квадратных скобках стоит интеграл от $\left|z-\mathbf{P}_{3}^{j}(h)\right|^{2}$ по $B_{h}^{j}$ ). При учете соотношений (2.39) и (2.35) получаем, что

$$
\left|a_{6-i}\right|^{2} \leqslant c h^{-10} h^{3} h^{2} \int_{B_{h}^{j}}\left(\left|u_{i}\right|^{2}+\left|v_{i}\right|^{2}\right) d x \leqslant c h^{-5}\left(h^{2}|\log h|^{2}\left|u ; \Omega_{h}^{0}\right|_{0}^{2}+h^{2} \mathscr{E}\left(u ; B_{h}^{j}\right)\right) .
$$

Доказательство леммы закончено.

\section{§3. Неравенства Корна для сочленений}

3.1. Основной трюк. Сузим поле $u \in \stackrel{\circ}{H^{1}}\left(\Omega(h) ; \Gamma^{0}(h)\right)^{3}$ на пластину $\Omega_{h}^{0}$ и представим его в виде

$$
u(y, z)=v(y, z)+d(y, z) a,
$$

где $a \in \mathbb{R}^{6}$, а слагаемое $v$ подчинено условию ортогональности (2.2). Поскольку для составляющей $v$ справедливо неравенство (2.3), достаточно вывести удовлетворительную оценку столбца $a$ - как и в доказательстве леммы 2.5 , элементарные операции с жестким смещением $d(y, z) a$ предопределяют структуру нормы $\|\cdot\|_{0}$ в неравенстве Корна (1.8).

Используем представление (2.1) для образования системы линейных уравнений для столбца $a=\left(a_{1}, \ldots, a_{6}\right)^{\top}$. Опишем один из способов: умножив (3.1) на $(6 \times 3)$-матрицу $d(y, z)^{\top}$, проинтегрируем результат по шару $B_{h}^{j} \subset g_{h}^{j}$ и получим равенство

$$
M^{j}(h) a=F^{j},
$$

в котором $(6 \times 6)$-матрица $M^{j}(h)$ и столбец $F^{j} \in \mathbb{R}^{6}$ имеют вид:

$$
\begin{gathered}
M^{j}(h)=\int_{B_{h}^{j}} d(y, z)^{\top} d(y, z) d y d z, \\
F^{j}=\int_{B_{h}^{j}} d(y, z)^{\top}(u(y, z)-v(y, z)) d y d z .
\end{gathered}
$$

Столбец (3.4) обрабатывается согласно леммам 2.5 и $2.7,2.8$, причем при оценке $u$ используется включение $B_{h}^{j} \subset G_{h}^{j}$, а при оценке $v$ - включение $B_{h}^{j} \subset \Omega_{h}^{0}$. Матрица (3.3) является матрицей Грама, построенной по столбцам матрицы (2.1) при 
помощи скалярного произведения в $L_{2}\left(B_{h}^{j}\right)^{3}$, т.е. $M^{j}(h)$ - симметрическая и положительно определенная матрица,

$$
\left\|M^{j}(h) ; \mathbb{R}^{6} \rightarrow \mathbb{R}^{6}\right\| \leqslant c \operatorname{mes}_{3} B_{h}^{j} \leqslant c h^{3}
$$

Тем не менее предел $h^{-3} M^{j}(h)$ при $h \rightarrow+0$ становится вырожденной матрицей и, следовательно, система (3.2) с фиксированным $j$ не может обслужить многие геометрические ситуации. Поэтому далее используется прием суммирования равенств (3.2) по $j=1, \ldots, J$.

Изложенная схема подходит для стола с достаточным количеством ножек $(J \geqslant 3)$. Если ножек одна или две, то приходится пользоваться явной формулой для матрицы (3.3), благо интегралы по шару вычисляются легко. Наконец, при обилии ножек $(J \geqslant 6)$ вместо (3.2) имеем дело с системами

$$
\mathscr{M}^{j}(h) a=\mathscr{F}^{j},
$$

при этом матрица и правая часть задаются формулами

$$
\begin{gathered}
\mathscr{M}^{j}(h)=\int_{B_{h}^{j}} d(y, z)^{\top} \mathbf{l}^{j}\left(\mathbf{l}^{j}\right)^{\top} d(y, z) d y d z, \\
\mathscr{F}^{j}=\int_{B_{h}^{j}} d(y, z)^{\top} \mathbf{1}^{j}\left(\mathbf{l}^{j}\right)^{\top}(u(y, z)-v(y, z)) d y d z,
\end{gathered}
$$

где $\mathbf{l}^{j}$ - единичный вектор (столбец) оси $\Lambda^{j}$. Способ вывода системы (3.5) остается прежним, но множителем берется $(6 \times 3)$-матрица $d(y, z)^{\top} \mathbf{l}^{j}\left(\mathbf{l}^{j}\right)^{\top}$. Выбор столбца $\mathbf{l}^{j}$ вполне понятен: в отличие от поперечных $u_{1}^{j}$ и $u_{2}^{j}$ продольная компонента $u_{3}^{j}=\left(\mathbf{1}^{j}\right)^{\top} u$ входит в нормы (2.9) и (2.22) без дополнительного множителя $h^{2}$. Подчеркнем, что матрица (3.6) изначально оказывается вырожденной.

Напоминаем, что в этом параграфе случай мязких стержней, вставленных в $n a з ы$, не обсуждается (см. далее п. 4.2).

3.2. Один стержень. Рассмотрим стержень $G_{h}^{1}$ и шар $B_{h}^{1} \subset g_{h}^{1}$ с центром $\mathbf{P}^{1}(h)$. Для упрошения вькладок перенесем начало декартовых координат в точку $\mathbf{P}^{1}(h)$, сохранив направления осей. Обозначив через $(\mathbf{y}, \mathbf{z})$ введенные координаты, при помощи простых вычислений (см. [18; 2.1$])$ находим, что

$$
\begin{gathered}
d(y, z) a=d(\mathbf{y}, \mathbf{z}) \mathbf{a}, \\
\mathbf{a}^{\prime}=a^{\prime}+d^{\prime \prime}\left(\mathbf{P}^{1}(h)\right) a^{\prime \prime}, \quad \mathbf{a}^{\prime \prime}=a^{\prime \prime} .
\end{gathered}
$$

Здесь $d^{\prime \prime}(y, z)$-правый $(3 \times 3)$-блок матрицы $(2.1)$. Так как изначально точку $x=0$ можно расположить на расстоянии $O(h)$ от $\mathbf{P}^{1}(h)$, согласно $(3.9)$ и $(2.1)$ имеем

$$
\left|a^{\prime}\right| \leqslant\left|\mathbf{a}^{\prime}\right|+c h\left|\mathbf{a}^{\prime \prime}\right|
$$


Кроме того, обозначив $w=d a$ и $\|\cdot\|_{0}=\left\|\cdot ; L_{2}\left(\Omega_{h}^{0}\right)\right\|$, видим, что

$$
\begin{aligned}
& \left\|w_{i}\right\|_{0}^{2} \leqslant \operatorname{ch}\left(\left|a_{i}\right|^{2}+h^{2}\left|a_{6-i}\right|^{2}+\left|a_{6}\right|^{2}\right), \quad i=1,2, \\
& \left\|w_{3}\right\|_{0}^{2} \leqslant \operatorname{ch}\left(\left|a_{3}\right|^{2}+\left|a_{4}\right|^{2}+\left|a_{5}\right|^{2}\right), \\
& \left\|\frac{\partial w_{1}}{\partial y_{2}}\right\|_{0}^{2}+\left\|\frac{\partial w_{2}}{\partial y_{1}}\right\|_{0}^{2} \leqslant c h\left|a_{6}\right|^{2}, \\
& \left\|\frac{\partial w_{i}}{\partial z}\right\|_{0}^{2}+\left\|\frac{\partial w_{3}}{\partial y_{i}}\right\|_{0}^{2} \leqslant \operatorname{ch}\left|a_{6-i}\right|^{2}, \quad i=1,2,
\end{aligned}
$$

а производные $\partial w_{k} / \partial x_{k}$ равны нулю (см. замечание 2.2$)$.

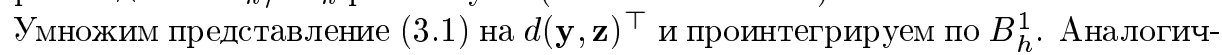
ные (3.2)-(3.4) формулы выглядят так:

$$
\begin{aligned}
& \mathbf{M}^{1}(h) \mathbf{a}=\mathbf{F}^{1}, \\
& \mathbf{M}^{1}(h)=\int_{B_{h}^{1}} d(\mathbf{y}, \mathbf{z})^{\top} d(\mathbf{y}, \mathbf{z}) d \mathbf{y} d \mathbf{z}, \\
& \mathbf{F}^{1}=\int_{B_{h}^{1}} d(\mathbf{y}, \mathbf{z})^{\top}(u(y, z)-v(y, z)) d \mathbf{y} d \mathbf{z} .
\end{aligned}
$$

Благодаря выбору области интегрирования матрица (3.13) становится диагональной и легко вычисляется:

$$
\mathbf{M}^{1}(h)=\frac{4}{3} \pi h^{3} R_{1}^{3} \operatorname{diag}\left\{1,1,1, \frac{3}{5} h^{2} R_{1}^{2}, \frac{3}{5} h^{2} R_{1}^{2}, \frac{3}{5} h^{2} R_{1}^{2}\right\} .
$$

Обрашаясь к формулам (3.12), (3.14), (3.15) и неравенствам $(2.39),(2.40)$ и (2.20), (2.31), обрабатываем компоненты столбца а:

$$
\begin{aligned}
\left|\mathbf{a}_{i}\right|^{2} \leqslant & c h^{-6}\left|\mathbf{F}_{i}^{1}\right|^{2} \leqslant c h^{-6} \operatorname{mes}_{3} B_{h}^{1} \int\left(\left|u_{i}\right|^{2}+\left|v_{i}\right|^{2}\right) d x \\
\leqslant & c h^{-3}\left(h^{-1} \mu_{3}(h)^{-1} \mathscr{E}_{\gamma}\left(u ; G_{h}^{1}\right)+h^{2}|\log h|^{2} \mathscr{E}\left(u ; \Omega_{h}^{0}\right)\right) \\
\leqslant & c h^{-4} \lambda_{3}(h)^{-1} \mathscr{E}_{\gamma}(u ; \Omega(h)) \\
\left|\mathbf{a}_{3}\right|^{2} \leqslant & c h^{-3} \int\left(\left|u_{3}\right|^{2}+\left|v_{3}\right|^{2}\right) d x \leqslant c h^{-3}\left(h^{-1} \mu_{3}(h)^{-1} \mathscr{E}_{\gamma}\left(u ; G_{h}^{1}\right)\right. \\
& \left.\quad+|\log h|^{2} \mathscr{E}\left(u ; \Omega_{h}^{0}\right)\right) \leqslant c h^{-4} \lambda_{1}(h)^{-1} \mathscr{E}_{\gamma}(u ; \Omega(h)) \\
\left|\mathbf{a}_{6-i}\right|^{2} \leqslant & c h^{-10}\left|\mathbf{F}_{6-i}^{1}\right|^{2} \\
\leqslant & c h^{-10} \sup _{B_{h}^{1}}\left|d^{\prime \prime}(\mathbf{y}, z)\right|^{2} \operatorname{mes}_{3} B_{h}^{1} \int \sum_{k=i, 3}\left(\left|u_{k}-\bar{u}_{k}\right|^{2}+\left|v_{k}-\bar{v}_{k}\right|^{2}\right) d x \\
\leqslant & c h^{-5}\left(h \mu_{1}(h)^{-1}+h^{2}|\log h|^{2}\right) \mathscr{E}_{\gamma}(u ; \Omega(h)) \leqslant c h^{-4} \lambda_{1}(h)^{-1} \mathscr{E}_{\gamma}(u ; \Omega(h)) \\
\left|\mathbf{a}_{6}\right|^{2} \leqslant & c h^{-5} \int \sum_{i=1}^{2}\left(\left|u_{i}-\bar{u}_{i}\right|^{2}+\left|v_{i}\right|^{2}\right) d x \\
\leqslant & c h^{-5}\left(h \mu_{1}(h)^{-1}+h^{2}|\log h|^{2}\right) \mathscr{E}_{\gamma}(u ; \Omega(h)) \leqslant c h^{-4} \lambda_{1}(h)^{-1} \mathscr{E}_{\gamma}(u ; \Omega(h))
\end{aligned}
$$


Здесь $i=1,2$, интегрирование ведется по шару $B_{h}^{1}, \mu_{k}(h)$ и $\lambda_{k}(h)$ - величины $(2.23)$ и (1.10), а в (3.18), (3.19) фигурируют средние (2.32).

Принимая во внимание соотношения (3.8)-(3.11) и (3.15), подбираем норму $\|\cdot\|_{0}$ так, чтобы обеспечить выполнение неравенства

$$
\left\|w ; \Omega_{h}^{0}\right\|_{0}^{2} \leqslant c \mathscr{E}_{\gamma}(u ; \Omega(h))
$$

Простые вычисления показывают, что нужно взять

$$
\begin{gathered}
\left\|u ; \Omega_{h}^{0}\right\|_{0}=\left(\int _ { \Omega _ { h } ^ { 0 } } \left(\sum_{i=1}^{2}\left(\left|\frac{\partial u_{i}}{\partial y_{i}}\right|^{2}+h^{3} \lambda_{1}(h)\left(\left|\frac{\partial u_{i}}{\partial z}\right|^{2}+\left|\frac{\partial u_{3}}{\partial y_{i}}\right|^{2}\right)+h^{3} \lambda_{1}(h)\left|u_{i}\right|^{2}\right)\right.\right. \\
\left.\left.+\left|\frac{\partial u_{3}}{\partial z}\right|^{2}+h^{3} \lambda_{1}(h)\left(\left|\frac{\partial u_{1}}{\partial y_{2}}\right|^{2}+\left|\frac{\partial u_{2}}{\partial y_{1}}\right|^{2}\right)+h^{3} \lambda_{1}(h)\left|u_{3}\right|^{2}\right) d x\right)^{1 / 2}
\end{gathered}
$$

(Пояснение: при выборе множителя у $\left|u_{i}\right|^{2}$ учтено, что $\lambda_{3}(h) \geqslant \lambda_{1}(h)$.) Заметив, что $\left\|v ; \Omega_{h}^{0}\right\|_{0} \leqslant\left|v ; \Omega_{h}^{0}\right|_{0}$, совмешаем оценки $(3.20),(2.3),(2.20)$ и формулируем результат.

ТеОрема 3.1. Справедливо неравенство Корна (1.8), в котором $\|\cdot\|_{0} u$ $\|\cdot\|_{j}-$ нормиц (3.21) и (2.22).

ЗАмечАние 3.2. Норма (3.21) эквивалентна

$$
\left(\mathscr{E}\left(u ; \Omega_{h}^{0}\right)+h^{3} \lambda_{1}(h)\left\|u ; H^{1}\left(\Omega_{h}^{0}\right)\right\|^{2}\right)^{1 / 2} .
$$

Опишем конкретные конструкции, подтверждающие асимптотическую точность неравенства Корна из теоремы 3.1 .

ПримеР 3.3. 1) Гриб. Пусть

$$
\Omega_{h}^{0}=\left\{x:|y|<R_{0},|z|<\frac{h}{2}\right\}, \quad G_{h}^{1}=\{x:|y|<h, z \in(-L, 0)\}
$$

и соответственно $P^{1}(h)=(0,0,-h / 4)^{\top}, R_{1}=1 / 4$. Рассмотрим поля

$$
\begin{aligned}
V^{0}(x) & =\left(y_{1} \mathbf{e}_{2}-y_{2} \mathbf{e}_{1}\right)\left(1-\chi\left(h^{-1}|y|\right)\right) \\
U^{0}(x) & =\left(y_{1} \mathbf{e}_{2}-y_{2} \mathbf{e}_{1}\right) X\left(-L^{-1} z\right) \\
V^{i}(x) & =y_{i} \mathbf{e}_{3}\left(1-\chi\left(h^{-1}|y|\right)\right)-z \mathbf{e}_{i} \frac{\partial}{\partial y_{i}}\left(y_{i}\left(1-\chi\left(h^{-1}|y|\right)\right)\right) \\
U^{i}(x) & =z \mathbf{e}_{i} X\left(-L^{-1} z\right)-y_{i} \mathbf{e}_{3} \frac{\partial}{\partial z}\left(z X\left(-L^{-1} z\right)\right)
\end{aligned}
$$

где $\mathbf{e}_{k}$ - орт оси $x_{k}, \chi$ и $X$ - срезаюшие функции из $C^{\infty}(\mathbb{R})$, причем $\chi(t)=1$ при $|t| \leqslant 1, \chi(t)=0$ при $|t| \geqslant 2$ и $X(t)=1$ при $t \leqslant 1 / 2, X(t)=0$ при $t \geqslant 1$. Заметим, что $U^{q}$ - жесткие смешения (повороты) на $\Omega_{h}^{0}$ и $V^{q}=0$ на $G_{h}^{1}$. Поэтому, рассчитьвая деформации $\varepsilon_{i k}\left(U^{q}\right)$ и $\varepsilon_{i k}\left(V^{q}\right)$ по формулам (1.7), обнаруживаем, что 
они (деформации) локализуются на множествах $\{x:|y|<h, z \in[-L,-L / 2]\}$ и $\{x: h \leqslant|y| \leqslant 2,|z| \leqslant h / 2\} ;$ к тому же

$$
\begin{aligned}
& \mathscr{E}_{\gamma}\left(U^{q} ; \Omega(h)\right) \leqslant c \gamma(h) h^{4}, \\
& \mathscr{E}_{\gamma}\left(V^{q} ; \Omega(h)\right) \leqslant c h^{3} .
\end{aligned}
$$

При $W^{q}=U^{q}$ и $W^{q}=V^{q}$ имеем

$$
\begin{aligned}
& \left\|W^{0} ; \Omega_{h}^{0}\right\|_{0}^{2} \geqslant h^{3} \lambda_{1}(h)\left(\left\|\frac{\partial W_{1}^{0}}{\partial y_{2}}\right\|_{0}^{2}+\left\|\frac{\partial W_{2}^{0}}{\partial y_{1}}\right\|_{0}^{2}+\left\|W_{1}^{0}\right\|_{0}^{2}+\left\|W_{2}^{0}\right\|_{0}^{2}\right) \geqslant c_{0} h^{4} \lambda_{1}(h), \\
& \left\|W^{i} ; \Omega_{h}^{0}\right\|_{0}^{2} \geqslant h^{3} \lambda_{1}(h)\left(\left\|\frac{\partial W_{i}^{i}}{\partial z}\right\|_{0}^{2}+\left\|\frac{\partial W_{3}^{i}}{\partial y_{i}}\right\|_{0}^{2}+\left\|W_{3}^{i}\right\|_{0}^{2}\right) \geqslant c_{i} h^{4} \lambda_{1}(h)
\end{aligned}
$$

здесь $c_{q}$ - положительные постоянные и $\|\cdot\|_{0}=\left\|\cdot ; L_{2}\left(\Omega_{h}^{0}\right)\right\|$. Итак, для "не очень жестких" стержней (т.е. при $\gamma(h) \leqslant \gamma_{1} h^{-1}|\log h|^{-2}$ и $\left.\lambda_{1}(h) \sim \gamma(h)\right)$ асимптотическая точность доказанного неравенства Корна устанавливается сравнением формул (3.26) и (3.28) с $W^{q}=U^{q}$, а для жестких стержней (т.е. при

$$
\gamma(h) \geqslant \gamma_{1} h^{-1}|\log h|, \quad \gamma_{1}>0
$$

и соответственно $\left.\lambda_{1}(h) \sim h^{-1}|\log h|^{-2}\right)$ - сравнением формул (3.27) и (3.28) с $W^{q}=V^{q}$. Как обычно (см., например, [5] и [18; § 3.1]), конструкции (3.22), (3.24), используюшие врашательные жесткие смешения, не позволяют подтвердить наличие $\log h$ в неравенстве Корна - к этому вопросу возвратимся в примере 3.7 .

2) Опахало. Пусть

$$
\begin{aligned}
& \Omega_{h}^{0}=\left\{x:\left|y_{1}+R_{0}\right|^{2}+y_{2}^{2}<R_{0}^{2},|z|<\frac{h}{2}\right\}, \\
& G_{h}^{1}=\left\{x:\left|y_{2}\right|^{2}+|z|^{2}<h^{2}, y_{1} \in(-h, L-h)\right\},
\end{aligned}
$$

при этом $z^{1}=y_{1}+h, y^{1}=\left(y_{2}, z\right)$ и $P^{1}(h)=(-h / 2,0,0)^{\top}, R_{1}=1 / 4$. Для проверки правильности расстановки весов в норме (3.21) опять-таки можно взять поля (3.22), (3.24), продолженные нулем на “жесткую” ручку $G_{h}^{1}$. В случае "не очень жесткой" ручки поля $(3.23),(3.25)$, используемые для названной цели, видоизменяются:

$$
\begin{aligned}
U^{0}(x) & =y_{1} \mathbf{e}_{2} X\left(L^{-1} y_{1}\right)-y_{2} \mathbf{e}_{1} \frac{\partial}{\partial y_{1}}\left(y_{1} X\left(L^{-1} y_{1}\right)\right) \\
U^{1}(x) & =y_{1} \mathbf{e}_{3} X\left(L^{-1} y_{1}\right)-z \mathbf{e}_{1} \frac{\partial}{\partial y_{1}}\left(y_{1} X\left(L^{-1} y_{1}\right)\right) \\
U^{2}(x) & =\left(z \mathbf{e}_{2}-y_{2} \mathbf{e}_{3}\right) X\left(L^{-1} y_{1}\right) .
\end{aligned}
$$


3.3. Два стержня. Пусть $J \geqslant 2$ и $P^{1} \neq P^{2}$, т.е. точки присоединения стержней к пластине удалены одна от другой. Совместим начало координат с точкой $P^{1}$, а ось $x_{1}=y_{1}$ направим от $P^{1}$ к $P^{2}$. Воспользуемся прежними формулами (3.8), $(3.9),(3.12)-(3.14)$ и оценками $(3.10),(3.11),(3.16)-(3.19)$, но при учете второго стержня улучшим оценку компоненты а 6 столбца а. Именно, поступая аналогично (3.5)-(3.7), умножим представление (3.1) на $d(\mathbf{y}, \mathbf{z})^{\top} \mathbf{e}_{1} \mathbf{e}_{1}^{\top}$ и проинтегрируем по шару $B_{h}^{2} \subset g_{h}^{2}$. Согласно (2.1) шестая строка полученной системы выглядит так:

$$
\begin{gathered}
\mathscr{M}_{61}^{2}(h) \mathbf{a}_{1}+\mathscr{M}_{66}^{2}(h) \mathbf{a}_{6}=-\int_{B_{h}^{2}} \mathbf{y}_{2}\left(u_{1}-v_{1}\right) d x \\
\left|\mathscr{M}_{61}^{2}(h)+\frac{4}{3} \pi h^{3} R_{2}^{3} P_{2}^{2}(0)\right| \leqslant c h^{4}, \quad\left|\mathscr{M}_{66}^{2}(h)-\frac{4}{3} \pi h^{3} R_{2}^{3} P_{2}^{2}(0)^{2}\right| \leqslant c h^{4}
\end{gathered}
$$

(воспользовались тем, что $\mathbf{y}_{2}=P_{2}^{2}+O(h)$ на $\left.B_{h}^{2}\right)$. Следовательно, оценивая интеграл из (3.32) аналогично (3.16) и применяя саму формулу (3.16), получаем соотношение

$$
\begin{aligned}
\left|\mathbf{a}_{6}\right|^{2} & \leqslant c\left(\left|\mathbf{a}_{1}\right|^{2}+c h^{-3} \int_{B_{h}^{2}} \mathbf{y}_{2}^{2}\left(\left|u_{1}\right|^{2}+\left|v_{1}\right|^{2}\right) d x\right) \\
& \left.\leqslant c\left(h^{-4} \lambda_{3}(h)^{-1}+h^{-3}\left(h^{-1} \mu_{3}(h)^{-1}+h^{2}|\log h|^{2}\right]\right)\right) \mathscr{E}_{\gamma}(u ; \Omega(h)) \\
& \leqslant c h^{-4} \lambda_{3}(h)^{-1} \mathscr{E}_{\gamma}(u ; \Omega(h)),
\end{aligned}
$$

которое в соответствии с (3.11) позволяет увеличить до $h^{3} \lambda_{3}(h)$ множители при $\left|\partial u_{1} / \partial y_{2}\right|^{2},\left|\partial u_{2} / \partial y_{1}\right|^{2}$ и $\left|u_{i}\right|^{2}$ в норме (3.21):

$$
\begin{aligned}
\left\|u ; \Omega_{h}^{0}\right\|_{0}= & \left(\int _ { \Omega _ { h } ^ { 0 } } \left(\sum_{i=1}^{2}\left(\left|\frac{\partial u_{i}}{\partial y_{i}}\right|^{2}+h^{3} \lambda_{1}(h)\left(\left|\frac{\partial u_{i}}{\partial z}\right|^{2}+\left|\frac{\partial u_{3}}{\partial y_{i}}\right|^{2}\right)+h^{3} \lambda_{3}(h)\left|u_{i}\right|^{2}\right)\right.\right. \\
& \left.\left.+\left|\frac{\partial u_{3}}{\partial z}\right|^{2}+h^{3} \lambda_{3}(h)\left(\left|\frac{\partial u_{1}}{\partial y_{2}}\right|^{2}+\left|\frac{\partial u_{2}}{\partial y_{1}}\right|^{2}\right)+h^{3} \lambda_{1}(h)\left|u_{3}\right|^{2}\right)\right)^{1 / 2},
\end{aligned}
$$

сохранив при этом неравенство (3.20). По-прежнему, $\left\|v ; \Omega_{h}^{0}\right\|_{0} \leqslant\left|v ; \Omega_{h}^{0}\right|_{0}$ и, следовательно, доказана

Теорема 3.4. Если $J \geqslant 2$ и $P^{1} \neq P^{2}$, то справедливо неравенство Корна (1.8), в котором $\|\cdot\|_{0} u\|\cdot\|_{j}-$ норми (3.34) u (2.22).

Замечание 3.5. Пусть стержень “очень жесткий”, т.е.

$$
\gamma(h) \geqslant \gamma_{3} h^{-3}|\log h|^{-2}, \quad \gamma_{3}>0,
$$

и соответственно $\lambda_{1}(h) \sim h^{-1}|\log h|^{-2}, \lambda_{3}(h) \sim h^{-3}|\log h|^{-2}$. Тогда норма (3.34) лишь дополнительными логарифмическими множителями отличается от нормы (2.4), а асимптотическая точность неравенства Корна проверяется при помоши анзаца, принятого в теории тонких пластин (см., например, [18; гл. 4]),

$$
W(x)=h^{-1} w_{3}(y)+\sum_{i=1}^{2}\left(w_{i}(y)-z \frac{\partial w_{3}}{\partial y_{i}}(y)\right) .
$$


Считая, что функции $w_{q} \in C^{\infty}\left(\bar{\omega}^{0}\right)$ аннулируются вблизи точек $P^{1}(0), \ldots, P^{J}(0)$, продолжаем поле (3.36) нулем на стержни и находим, что

$$
\mathscr{E}_{\gamma}(W ; \Omega(h))=\mathscr{E}\left(W ; \Omega_{h}^{0}\right) \leqslant c h .
$$

При выполнении условия (3.35) все слагаемые из подынтегрального выражения в (3.34), за исключением $\left|\partial u_{3} / \partial z\right|^{2}$ (ср. с концом доказательства леммы 2.1), придают квадрату нормы $\left\|u ; \Omega_{h}^{0}\right\|_{0}^{2}$ порядок $h|\log h|^{-2}$ (по поводу $\log h$ см. далее пример 3.7).

В следующих примерах рассматриваем только случай $\gamma(h) \leqslant \gamma_{3} h^{-3}|\log h|^{-2}$.

ПримеР 3.6. 1) Парковая скамейка. Пусть

$$
\begin{gathered}
\Omega_{h}^{0}=\left\{x:\left|y_{1}\right|<2,\left|y_{2}\right|<\frac{1}{2},|z|<\frac{h}{2}\right\}, \\
G_{h}^{j}=\left\{x:\left|y_{1}-(-1)^{j}\right|^{2}+\left|y_{2}\right|^{2} \leqslant h^{2}, z \in(-L, 0)\right\}, \quad j=1,2,
\end{gathered}
$$

при этом $P^{1}(h)=(-1,0,-h / 2)^{\top}$ и $P^{2}(h)=(1,0,-h / 2)^{\top}$. В случае "не очень мягких" стержней для проверки асимптотической точности неравенства Корна из теоремы 3.4 можно привлечь поле $\mathbf{U}^{0}=U^{2}$ из $(3.25)$ и поля

$$
\begin{aligned}
& \mathbf{U}^{1}(x)= \begin{cases}\mathbf{e}_{1}, & x \in \Omega_{h}^{0} ; \\
\mathbf{e}_{1} \chi\left(-L^{-1} z\right)-\left(y_{1}-P_{1}^{j}(0)\right) \mathbf{e}_{3} \frac{\partial}{\partial z} \chi\left(-L^{-1} z\right), & x \in G_{h}^{j},\end{cases} \\
& \mathbf{U}^{2}(x)=\mathbf{e}^{2} \chi\left(-L^{-1} z\right)-y_{2} \mathbf{e}_{3} \frac{\partial}{\partial z} \chi\left(-L^{-1} z\right) .
\end{aligned}
$$

Отметим, что $\mathscr{E}_{\gamma}\left(\mathbf{U}^{q} ; \Omega(h)\right) \leqslant c \gamma(h) h^{4}$ (cp. (3.26)) и при расположении осей ножек в плоскости $\left\{x: y_{1}=0\right\}$ вместо $U^{2}$ берется поле $U^{1}$.

Если $\gamma_{1} h^{-1}|\log h|^{-2} \leqslant \gamma(h) \leqslant \gamma_{3} h^{-3}|\log h|^{-2}$ (см. (3.30) и (3.35)), то для подтверждения правильности выбора весовых множителей при $\left|u_{i}\right|^{2}$ и $\left|u_{3}\right|^{2}$ подходят поля $\mathbf{U}^{i}$ из (3.37) и $\mathbf{V}^{2}=V^{2}$ из (3.24) соответственно.

2) Волокуши. Пусть $P^{1}(h)=(0,1-h / 2,0)^{\top}, P^{2}(h)=(0,-1+h / 2,0)^{\top}$ и

$$
\begin{gathered}
\Omega_{h}^{0}=\left\{x: y_{1} \in(-2,0),\left|y_{2}\right|<1\right\}, \\
G_{h}^{j}=\left\{x:\left|y_{2}-(-1)^{j}(1-h)\right|^{2}+|z|^{2}<h^{2}, y_{1} \in(-h, L-h)\right\}, \quad j=1,2 .
\end{gathered}
$$

“Оглобли" $G_{h}^{1}$ и $G_{h}^{2}$ лежат в той же плоскости, что и “настил" $\Omega_{h}^{0}$, однако поля (3.37) переделываются в нужные так же, как поля (3.22)-(3.25) - в поля (3.31).

Приведенные конкретные векторы смешений демонстрируют, что добавлениев-ряд ножек у скамейки и оглоблей у волокуш не увеличивают весовые множители в (3.34), а значит, не усиливают конструкцию - эти факты, разумеется, известны из повседневной жизни. В следующих пунктах будет проверено, что правильное размешение дополнительных стержней позволяет все-таки улучшить норму (3.34). 
ПРИмеР 3.7. Логарифмы. В рамках примера 3.6.1) положим

$$
\mathscr{V}^{i}(h, x)=\mathbf{e}_{i} X\left(\frac{\log \left|y^{1}\right|}{\log h}\right) X\left(\frac{\log \left|y^{2}\right|}{\log h}\right), \quad i=1,2,
$$

где $y^{j}=y-P^{i}(0)$, а $X$ - срезаюшая функция, использованная в примере 3.3 .1$)$. Ясно, что при малом $h$

$$
\left\|\mathscr{Y}_{i}^{i} ; L_{2}\left(\Omega_{h}^{0}\right)\right\|^{2} \geqslant c_{0} h, \quad c_{0}>0 .
$$

Деформации $\varepsilon_{p q}\left(\mathscr{V}^{i}\right)$ содержат производные срезок, а значит, их носители совпадают с объединением колец $\left\{x: h \leqslant\left|y^{j}\right| \leqslant h^{1 / 2},|z| \leqslant h / 2\right\}$, на которых $\left|\varepsilon_{p q}\left(\mathscr{V}^{i} ; x\right)\right| \leqslant c|\log h|^{-1}\left|y^{j}\right|^{-1}$. Таким образом,

$$
\begin{aligned}
\mathscr{E}\left(\mathscr{V}^{i} ; \Omega_{h}^{0}\right) & \leqslant 2 C \int_{-h / 2}^{h / 2} d z \int_{h}^{h^{1 / 2}}|\log h|^{-2} r^{-2} r d r \\
& =\left.2 C h|\log h|^{-2} \log r\right|_{h} ^{h^{1 / 2}}=C h|\log h|^{-1}
\end{aligned}
$$

и необходимость присутствия логарифмических множителей в весовой норме (3.34) подтверждена.

3.4. Шесть стержней. В силу замечания 3.5 увеличение весовых множителей в норме (3.34) в общем случае невозможно и далее речь пойдет только об уменьшении границ в определениях (3.30) и (3.35) достаточной жесткости стержней.

Пусть $J \geqslant 6$. Сформулируем геометрическое требование:

3) линейная оболочка строк $\mathbf{d}^{1}, \ldots, \mathbf{d}^{J} \in\left(\mathbb{R}^{6}\right)^{\top}$,

$$
\mathbf{d}^{j}=\left(l^{j}\right)^{\top} d\left(P^{j}\right),
$$

совпадает с евклидовым пространством $\left(\mathbb{R}^{6}\right)^{\top}$.

Перенумеровав при необходимости точки, считаем, что оболочка строк $\mathbf{d}^{1}, \ldots$, $\mathbf{d}^{6}$ обладает нужньм свойством. Подчеркнем, что ограничение из теоремы 3.4 снято и среди точек $P^{1}, \ldots, P^{6}$ могут быть одинаковые (допускаются конструкции, фрагментами которых служат “треноги” или "козлы”).

Обозначим через $\mathscr{M}(h)$ и $\mathscr{F}$ суммы матриц (3.6) и столбцов $(3.7)$ по $j=1, \ldots, 6$. Верны соотношения

$$
\mathscr{M}^{j}(h)=h^{3}\left(\frac{4}{3} \pi R_{j}^{3}\left(\mathbf{d}^{j}\right)^{\top} \mathbf{d}^{j}+O(h)\right) .
$$

Матрица

$$
m=\frac{4}{3} \pi \sum_{j=1}^{6} R_{j}^{3}\left(\mathbf{d}^{j}\right)^{\top} \mathbf{d}^{j}
$$

симметрическая и положительно определенная. Более того, если $b^{\top} m b=0$ для некоторого столбца $b \in \mathbb{R}^{6}$, то ввиду предположения 3 )

$$
0=b^{\top} m b \geqslant \frac{4}{3} \pi \sum_{j=1}^{6} R_{j}^{3}\left|\mathbf{d}^{j} b\right|^{2} \Rightarrow \mathbf{d}^{1} b=\cdots=\mathbf{d}^{6} b=0 \Rightarrow b=0,
$$


т.е. матрица (3.40) положительно определена и в силу (3.39)

$$
\left\|\mathscr{M}(h)^{-1} ; \mathbb{R}^{6} \rightarrow \mathbb{R}^{6}\right\| \leqslant c h^{-3} .
$$

Займемся правой частью системы $\mathscr{M}(h) a=\mathscr{F}$. Так как $\left(\mathbf{1}^{j}\right)^{\top} u=u_{3}^{j}$, при помощи (2.20) и (2.39) получаем, что

$$
\begin{aligned}
\left\|\mathscr{F}^{j} ; \mathbb{R}^{6}\right\|^{2} \leqslant & c \operatorname{mes}_{3} B_{h}^{j} \int_{g_{h}^{j}}\left(\left|u_{3}^{j}\right|^{2}+|v|^{2}\right) d x \leqslant c h^{3}\left(h \mu_{1}(h)^{-1} \mathscr{E}_{\gamma}\left(u ; G_{h}^{j}\right)\right. \\
& \left.+|\log h|^{2} \mathscr{E}\left(u ; \Omega_{h}^{0}\right)\right) \leqslant c h^{4} \lambda_{-1}(h)^{-1} \mathscr{E}_{\gamma}(u ; \Omega(h)) .
\end{aligned}
$$

Итак, справедливо соотношение

$$
\left\|a ; \mathbb{R}^{6}\right\|^{2} \leqslant c h^{-6}\left\|\mathscr{F} ; \mathbb{R}^{6}\right\|^{2} \leqslant c h^{-2} \lambda_{-1}(h)^{-1} \mathscr{E}_{\gamma}(u ; \Omega(h)),
$$

которое улучшает оценки (3.17), (3.19) компонент $\mathbf{a}_{3}$ и $\mathbf{a}_{4}, \mathbf{a}_{5}$. Учитывая "старые" неравенства (3.16), (3.33) для $\mathbf{a}_{1}, \mathbf{a}_{2}, \mathbf{a}_{6}$, а также формулы $(3.10),(3.11)$ и $\lambda_{1}(h)^{-1} \leqslant \lambda_{-1}(h)^{-1}$, составляем норму

$$
\begin{aligned}
\left\|u ; \Omega_{h}^{0}\right\|_{0}= & \left(\int _ { \Omega _ { h } ^ { 0 } } \left(\sum_{i=1}^{2}\left(\left|\frac{\partial u_{i}}{\partial y_{i}}\right|^{2}+h \lambda_{-1}(h)\left(\left|\frac{\partial u_{i}}{\partial z}\right|^{2}+\left|\frac{\partial u_{3}}{\partial y_{i}}\right|^{2}\right)+h^{3} \lambda_{3}(h)\left|u_{i}\right|^{2}\right)\right.\right. \\
& \left.\left.+\left|\frac{\partial u_{3}}{\partial z}\right|^{2}+h^{3} \lambda_{3}(h)\left(\left|\frac{\partial u_{1}}{\partial y_{2}}\right|^{2}+\left|\frac{\partial u_{2}}{\partial y_{1}}\right|^{2}\right)+h \lambda_{-1}(h)\left|u_{3}\right|^{2}\right)\right)^{1 / 2},
\end{aligned}
$$

которая по-прежнему мажорируется нормой $\left|u ; \Omega_{h}^{0}\right|_{0}$ из (2.4).

ТЕОРема 3.8. Если $J \geqslant 6$ и выполнено условие 3 ), то справедливо неравенство Корна (1.8), в котором $\|\cdot\|_{0} u\|\cdot\|_{j}-$ нормы (3.44) u (2.22).

ЗАмЕчАнИЕ 3.9. Если стержни "не очень мягкие", т.е.

$$
\gamma(h) \geqslant \gamma_{-1} h, \quad \gamma_{-1}>0
$$

множители при $\left|\partial u_{3} / \partial y_{i}\right|^{2}$ и $\left|u_{3}\right|^{2}$ в норме (3.44) становятся равными $h^{2}|\log h|^{-2}$ и лишь логарифмами отличаются от подобных множителей в норме (2.4). Другими словами, даже слабые растяжки при правильном расположении позволяют максимально снизить просадку пластины.

ПримеР 3.10. Таракан. Пусть $\Omega_{h}^{0}$ - эллиптическая в плане пластина

$$
\left\{x: y_{1}^{2}+4 y_{2}^{2}<9,|z|<\frac{h}{2}\right\},
$$

к которой снизу присоединены шесть тонких стержней со следующими атрибутами $\left(\mathbf{1}^{j}\right)^{\top},\left(P^{j}\right)^{\top}$ :

$$
\begin{array}{lll}
j=1: & \left(0,-2^{-1 / 2},-2^{-1 / 2}\right), & \left(-2,-2^{-1}, 0\right) ; \\
j=2: & (0,0,-1), & (0,-1,0) ; \\
j=3: & \left(2^{-1},-2^{-1},-2^{-1 / 2}\right), & \left(2,-2^{-1}, 0\right) ; \\
j=4: & \left(2^{-1}, 2^{-1},-2^{-1 / 2}\right), & \left(2,2^{-1}, 0\right) ; \\
j=5: & (0,0,-1), & (0,1,0) ; \\
j=6: & \left(0,2^{-1 / 2},-2^{-1 / 2}\right), & \left(-2,2^{-1}, 0\right) .
\end{array}
$$

Громоздкие, но простые вычисления показывают, что геометрическое условие 3) вьполнено. 
3.5. Три стержня. Конкретизируя положение осей $\Lambda^{j}$, можно уменьшить количество стержней при сохранении утверждения теоремы 3.8 или изменить в (3.44) весовые множители при $\left|\partial u_{1} / \partial y_{2}\right|^{2},\left|\partial u_{2} / \partial y_{1}\right|^{2}$ и $\left|u_{i}\right|^{2}$.

Стол. Пусть $J \geqslant 3$. Предположим, что

4) среди стержней $G_{h}^{1}, \ldots, G_{h}^{J}$ найдутся три, направленных вертикально и присоединенных к пластине в вершинах невырожденного треугольника.

Соблюдая указанное требование и не ограничивая общности, считаем, что нужными свойствами обладают стержни $G_{h}^{1}, G_{h}^{2}$ и $G_{h}^{3}$, т.е.

$$
\begin{gathered}
\mathbf{l}^{1}=\mathbf{l}^{2}=\mathbf{l}^{3}=\mathbf{e}_{3}, \\
\text { точки } P^{1}, P^{2} \text { и } P^{3} \text { не лежат на одной прямой. }
\end{gathered}
$$

Воспользуемся оценками (3.16) и (3.33), но улучшим оценки компонент столбца $\widehat{\mathbf{a}}=\left(\mathbf{a}_{3}, \mathbf{a}_{4}, \mathbf{a}_{5}\right)^{\top}$. Фиксируем систему координат так же, как и в п. 3.3 ; в силу $(3.47)$ $P_{1}^{2}>0$ и $P_{2}^{3} \neq 0$. Поскольку согласно (3.46) элементы строк $\widehat{\mathbf{d}}^{1}, \widehat{\mathbf{d}}^{2}$ и $\widehat{\mathbf{d}}^{3}$ из (3.38) с номерами 1,2 и 6 нулевые, вырождаются строки и столбцы матриц $\mathscr{M}^{1}(h), \mathscr{M}^{2}(h)$ и $\mathscr{M}^{3}(h)$ с теми же номерами. Ненулевые $(3 \times 3)$-блоки матриц обозначим $\widehat{\mathscr{M}}^{1}(h)$, $\widehat{\mathscr{M}}^{2}(h)$ и $\widehat{\mathscr{M}}^{3}(h)$, а их сумму $\widehat{\mathscr{M}}(h)$. Заметив, что укороченные строки

$$
\widehat{\mathbf{d}}^{1}=(1,0,0), \quad \widehat{\mathbf{d}}^{2}=\left(1,0,-P_{1}^{2}\right), \quad \widehat{\mathbf{d}}^{3}=\left(1, P_{2}^{3},-P_{1}^{3}\right)
$$

линейно независимы, и повторив рассуждения, касающиеся матриц (3.39) и (3.40), проверяем аналогичное (3.41) неравенство

$$
\left\|\widehat{\mathscr{M}}(h)^{-1} ; \mathbb{R}^{3} \rightarrow \mathbb{R}^{3}\right\| \leqslant c h^{-3} .
$$

Получая из (3.41) нужную информацию о правой части системы $\widehat{\mathscr{M}}(h) \widehat{\mathbf{a}}=\widehat{\mathscr{F}}$, приходим к искомой оценке

$$
\left\|\widehat{\mathbf{a}} ; \mathbb{R}^{3}\right\|^{2} \leqslant c h^{-6}\left\|\widehat{\mathscr{F}} ; \mathbb{R}^{3}\right\|^{2} \leqslant c h^{-2} \lambda_{-1}(h)^{-1} \mathscr{E}_{\gamma}(u ; \Omega(h))
$$

и к норме (3.44), обеспечивающей соотношение (3.20) для жесткого смещения $w(y, z)=d(y, z) a$.

ТЕОРема 3.11. Если $J \geqslant 3$ и выполнено условие 4), то справедливо неравенство Корна (1.8), в котором $\|\cdot\|_{0} u\|\cdot\|_{j}-$ нормы (3.44) u (2.22).

В связи с проблемой [1; с. 293], упоминавшейся в п. 1.1, укажем, что увеличение количества вертикальных ножек у стола не может повлиять на весовые множители в норме (3.44).

Рулевое колесо. Считая, что по-прежнему $J \geqslant 3$, вводим новое геометрическое ограничение:

5) среди стержсей $G_{h}^{1}, \ldots, G_{h}^{J}$ найдутся три с осями $\Lambda^{j}$, лежащими в плоскости $\{x: z=0\}$, и точками присоединения $P^{j}$, являющимися вериинами невырожденного треугольника. 
Пусть провозглашенными свойствами обладают стержни $G_{h}^{1}, G_{h}^{2}$ и $G_{h}^{3}$. Обозначив через " " операцию вычеркивания компонент или строк и столбцов с номерами $3,4,5$, видим, что в силу условия 5) строки $\widetilde{\mathbf{d}}^{1}, \widetilde{\mathbf{d}}^{2}$ и $\widetilde{\mathbf{d}}^{3}$, найденные согласно (2.1) и (3.38), образуют базис в пространстве $\left(\mathbb{R}^{3}\right)^{\top}$. Таким образом, прежние аргументы, примененные к сумме $\widetilde{\mathscr{M}}(h)$ урезанных матриц $\widetilde{\mathscr{M}}^{1}(h), \widetilde{\mathscr{M}}^{2}(h)$ и $\widetilde{\mathscr{M}}^{3}(h)$, приводят к соотношению

$$
\left\|\widetilde{\mathscr{M}}(h)^{-1} ; \mathbb{R}^{3} \rightarrow \mathbb{R}^{3}\right\| \leqslant c h^{-3} .
$$

Учитывая условие 5) и структуру двух верхних строк матрицы (2.1), обнаруживаем, что элемент $\mathbf{a}_{3}$ отсутствует в системе

$$
\widetilde{\mathscr{M}}(h) \widetilde{\mathbf{a}}=\widetilde{\mathscr{F}}-\widetilde{m}^{1}(h) \widetilde{\mathbf{a}}_{4}-\widetilde{m}^{2}(h) \mathbf{a}_{5}
$$

и, более того, $\left\|\widetilde{m}^{i}(h) ; \mathbb{R}^{3} \rightarrow \mathbb{R}^{3}\right\| \leqslant c h^{4}$. Наконец,

$$
\begin{aligned}
\left\|\widetilde{\mathscr{F}}^{j} ; \mathbb{R}^{3}\right\|^{2} & \leqslant c h^{3} \int_{g_{h}^{j}}\left(\left|u_{3}^{j}\right|^{2}+\left|v_{1}\right|^{2}+\left|v_{2}\right|^{2}\right) d x \\
& \leqslant c h^{3}\left(h \mu_{1}(h)^{-1}+h^{2}|\log h|^{2}\right) \mathscr{E}_{\gamma}(u ; \Omega(h)) \leqslant c h^{4} \mu_{1}(h)^{-1} \mathscr{E}_{\gamma}(u ; \Omega(h))
\end{aligned}
$$

и при помощи (3.18) заключаем, что

$$
\begin{aligned}
\left\|\widetilde{\mathbf{a}} ; \mathbb{R}^{3}\right\|^{2} & \leqslant c h^{-6}\left(\left\|\widetilde{\mathscr{F}} ; \mathbb{R}^{3}\right\|^{2}+h^{8}\left(\left|\mathbf{a}_{4}\right|^{2}+\left|\mathbf{a}_{5}\right|^{2}\right)\right) \\
& \leqslant c h^{-6}\left(h^{4} \mu_{1}(h)^{-1}+h^{4} \lambda_{1}(h)^{-1}\right) \mathscr{E}_{\gamma}(u ; \Omega(h)) \leqslant c h^{-2} \lambda_{1}(h)^{-1} \mathscr{E}_{\gamma}(u ; \Omega(h)) .
\end{aligned}
$$

По обычной схеме эта оценка вместе с (3.10), (3.11) и (3.16), (3.18) преврашается в очередное утверждение.

ТЕОРема 3.12. Если $J \geqslant 3$ и выполнено требование 5), то справедливо неравенство Корна $(1.8)$, в котором $\|\cdot\|_{j}-$ норма (2.22) и

$$
\begin{aligned}
\left\|u ; \Omega_{h}^{0}\right\|_{0}=( & \int_{\Omega_{h}^{0}}\left(\sum_{i=1}^{2}\left(\left|\frac{\partial u_{i}}{\partial y_{i}}\right|^{2}+h^{3} \lambda_{1}(h)\left(\left|\frac{\partial u_{i}}{\partial z}\right|^{2}+\left|\frac{\partial u_{3}}{\partial y_{i}}\right|^{2}\right)+h \lambda_{1}(h)\left|u_{i}\right|^{2}\right)\right. \\
& \left.\left.+\left|\frac{\partial u_{3}}{\partial z}\right|^{2}+h \lambda_{1}(h)\left(\left|\frac{\partial u_{1}}{\partial y_{2}}\right|^{2}+\left|\frac{\partial u_{2}}{\partial y_{1}}\right|^{2}\right)+h^{3} \lambda_{1}(h)\left|u_{3}\right|^{2}\right)\right)^{1 / 2} .
\end{aligned}
$$

Подчеркнем, что в условиях теоремы 3.12 сказанное в замечании 3.5 сохраняет силу при ограничении (3.45), более слабом, чем (3.35).

Совместим результаты, установленные в теоремах 3.11 и 3.12 , и тем самым уточним теорему 3.8 .

СлЕДСТВИЕ 3.13. Пусть $J \geqslant 6$ и выполнены оба требования 4) и 5), обеспечивающие, в частности, и требование 3). Тогда справедливо неравенство Корна (1.8), в котором $\|\cdot\|_{j}-$ норма (2.22) и

$$
\begin{gathered}
\left\|u ; \Omega_{h}^{0}\right\|_{0}=\left(\int _ { \Omega _ { h } ^ { 0 } } \left(\sum_{i=1}^{2}\left(\left|\frac{\partial u_{i}}{\partial y_{i}}\right|^{2}+h^{3} \lambda_{-1}(h)\left(\left|\frac{\partial u_{i}}{\partial z}\right|^{2}+\left|\frac{\partial u_{3}}{\partial y_{i}}\right|^{2}\right)+h \lambda_{1}(h)\left|u_{i}\right|^{2}\right)\right.\right. \\
\left.\left.+\left|\frac{\partial u_{3}}{\partial z}\right|^{2}+h \lambda_{1}(h)\left(\left|\frac{\partial u_{1}}{\partial y_{2}}\right|^{2}+\left|\frac{\partial u_{2}}{\partial y_{1}}\right|^{2}\right)+h^{3} \lambda_{-1}(h)\left|u_{3}\right|^{2}\right)\right)^{1 / 2}
\end{gathered}
$$




\section{§4. Обсуждение и обобщения}

4.1. О форме пластины и стержней. Для упрошения изложения элементы сочленения $\Omega(h)$ предполагались цилиндрическими телами. Такое ограничение снимается при сохранении всех обозначений и результатов. Для этой цели подходит разработанный в [13], [14] прием предварительной проверки неравенства Корна на более тонком (например, цилиндрическом) теле и последующего переноса неравенства на исходное тело. Так, в [15], [16] (см. также [18; §§3.3, 3.4]) были рассмотрены слабо изогнутые стержни и пластины с переменными сечением и толщиной. Дополнительные идеи понадобились в [17] и в [18; §3.4] при выводе весовых анизотропных неравенств Корна для стержней и пластин с быстроосциллируюшими границами и ячейками периодичности произвольной формы. В то же время переход к сочленениям (1.3) с нецилиндрическими элементами загромождает доказательства техническими приемами, повторяющими использованные в цитированных публикациях и затемняющими существо исследования.

В п. 1.4 и п. 2.3 было продемонстрировано, как следует обходиться с пазами и концами стержней произвольной формы. Аналогичные возмушения цилиндрических гранищ допустимы и для кромки пластины, и для зашемленных торцов стержней (ср. с [18]).

4.2. Пластина с пазами. В $\S 3$ из рассмотрения были исключены сочленения с мягкими стержнями, заделанными в пазы. Причиной тому послужила невозможность использования в данном случае простой леммы 2.1 , обслуживающей те сочленения (1.3), в которых стержни не проникают в пластину или вьполняется условие $\gamma(h) \geqslant 1$, т.е. стержни не мягче, чем пластина, и $\mathscr{E}\left(u ; \Omega_{h}^{0}\right) \leqslant \mathscr{E}_{\gamma}(u ; \Omega(h))$. Здесь при ограничении 2) из п. 1.4 будет проверено неравенство Корна

$$
\left|v ; \Omega_{h}\right|_{0}^{2} \leqslant c \mathscr{E}\left(v ; \Omega_{h}\right)
$$

для пластины с пазами $\Omega_{h}=\Omega_{h}^{0} \backslash\left(G_{h}^{1} \cup \cdots \cup G_{h}^{J}\right)$. Вместо условия ортогональности (2.2) зададим условие

$$
\int_{\Pi_{h}} d(y, z)^{\top} v(y, z) d y d z=0 \in \mathbb{R}^{6},
$$

где $\Pi_{h}=\Sigma_{h}^{0} \times(-h / 2, h / 2)$, а $\Sigma_{R}^{0}-$ срединное сечение $\Sigma^{0}$ с вырезанными $R_{0}$-окрестностями точек $P^{j}$, причем размер $R_{0}>0$ подобран так, чтобы $g_{h}^{j} \not \subset \Pi_{h}$ при $h \in\left(0, h_{0}\right]$. Подчеркнем, что замена $(2.2)$ на (4.2) не сказьвается на доказательстве самой леммы 2.1 (использованное в (2.6) неравенство Корна на $\Omega_{1}^{0}$ верно и при условии ортогональности (2.7) с П 1 вместо $\Omega_{1}^{0}$; см., например, [5], [18] и др.); другими словами, все рассуждения из $\S 3$ сохраняют силу.

ЛЕмма 4.1. Для поля $v \in H^{1}\left(\Omega_{h}\right)^{3}$, подчиненного условию ортогональности (4.2), справедливо неравенство Корна (4.1) с анизотропной нормой (2.4) u постоянной $c$, не зависящей от и и $h \in\left(0, h_{0}\right]$. 
ДокАЗАТЕльство. Сужение $v^{j}$ поля $v$ на $\mathscr{K}_{h}^{j} \backslash g_{h}^{j}$ (см. конецп. 1.4) представим в виде

$$
v^{j}(x)=V^{j}(x)+d(x) A^{j},
$$

подчинив слагаемое $V^{j}$ аналогичному (2.17) условию ортогональности

$$
\int_{\mathscr{B}_{h}^{j}} d(x)^{\top} V^{j}(x) d x=0 \in \mathbb{R}^{6} .
$$

При помощи формул (1.13) и (2.18) с $\Xi_{h}^{p}=\mathscr{B}_{h}^{j}$ выводим цепочку неравенств

$$
\begin{aligned}
\left\|V^{j} ; \mathscr{K}_{h}^{j} \backslash g_{h}^{j}\right\|^{2} & \leqslant c\left(\mathscr{E}\left(V^{j} ; \mathscr{K}_{h}^{j} \backslash g_{h}^{j}\right)+\left\|V^{j} ; \mathscr{B}_{h}^{j}\right\|^{2}\right) \\
& \leqslant c\left(\mathscr{E}\left(v^{j} ; \mathscr{K}_{h}^{j} \backslash g_{h}^{j}\right)+\mathscr{E}\left(V^{j} ; \mathscr{B}_{h}^{j}\right)\right) \leqslant 2 c \mathscr{E}\left(v^{j} ; \mathscr{K}_{h}^{j} \backslash g_{h}^{j}\right) .
\end{aligned}
$$

Следовательно, вспоминая оператор продолжения $\beta^{j}$ и соотношение (1.14), обнаруживаем, что поле $v$, доопределенное на $g_{h}^{j}$ равенством

$$
v=\beta^{j} V^{j}+d A^{j},
$$

попадает в пространство $H^{1}\left(\Omega_{h}^{0}\right)^{3}$ и, более того, подчиняется оценке

$\mathscr{E}\left(v ; \Omega_{h}^{0}\right)=\mathscr{E}\left(v ; \Omega_{h}\right)+\sum_{j=1}^{J} \mathscr{E}\left(\beta^{j} V^{j} ; g_{h}^{j}\right) \leqslant \mathscr{E}\left(v ; \Omega_{h}\right)+c \sum_{j=1}^{J}\left\|\beta^{j} V^{j} ; g_{h}^{j}\right\|^{2} \leqslant c \mathscr{E}\left(v ; \Omega_{h}\right)$

(воспользовались тем, что $\varepsilon_{i k}(v)=\varepsilon_{i k}\left(\beta^{j} V^{j}\right)$ на $g_{h}^{j}$ согласно замечанию 2.2 ). Осталось применить лемму 2.1 к продолженному полю $v$ и затем превратить $(2.3)$ в (4.1), вернувшись к пластине с пазами.

Благодаря тому, что в п. 2.3 допускались концы стержней и пазы с достаточно произвольными формами, можно искусственно нарастить стержни малыми, диаметром $O(h)$, фрагментами пластины и тем самьм свести задачу к изученной ранее. Итак, при замене леммы 2.1 леммой 4.1 все теоремы из $\S 3$ сохраняют силу для сочленений с мягкими стержнями, проникающими в жесткую пластину.

4.3. Составной стержень. Рассмотрим цилиндрический (для простоты) стержень $G_{h}=\left\{x: y \in \omega, z \in\left(-L_{-}, L_{+}\right)\right\}$- обозначения взяты из п. 1.1, но индекс $j$ не пишется. Пусть стержни $G_{h}^{ \pm}=\left\{x \in G_{h}: \pm z>0\right\}$ имеют различаюшиеся упругие свойства и соответственно $\mathscr{E}_{\gamma}\left(u ; G_{h}\right)=\mathscr{E}\left(u ; G_{h}^{+}\right)+$ $\gamma(h) \mathscr{E}\left(u ; G_{h}^{-}\right)$. Аналогичная ситуация уже встречалась в п. 2.3 , где стержень $G_{h}^{+}$ имел малую длину $L_{+}=O(h)$. Вместе с тем, доказательство леммы 2.5 легко переносится на случай $L_{+}=O(1)$. Действительно, положим $g_{h}=\left\{x \in G_{h}\right.$ : $0<z<h\}$ и распространим представление $(2.33)$ с шара $B_{h} \subset g_{h}$ на весь стержень $G_{h}^{+}$. В силу неравенств (1.11) и (2.18) имеем

$$
\begin{aligned}
\left\|v ; g_{h}\right\|^{2} & \leqslant c\left(\mathscr{E}\left(v ; g_{h}\right)+\left\|v ; B_{h}\right\|^{2}\right) \leqslant c \mathscr{E}\left(u ; g_{h}\right), \\
\mathscr{E}\left(\chi_{h} v ; G_{h}^{+}\right) & \leqslant c\left(\mathscr{E}\left(v ; G_{h}^{+}\right)+h^{-2}\left\|v ; L_{2}\left(g_{h}\right)\right\|^{2}\right) \leqslant C \mathscr{E}\left(u ; G_{h}^{+}\right),
\end{aligned}
$$


здесь $\chi_{h}(z)=1-X\left(h^{-1} z\right)$ и $X$ - срезающая функция, введенная в примере 3.3 .1$)$. Так как $\chi_{h} v \in \stackrel{\circ}{H}{ }^{1}\left(G_{h}^{+} ; \Gamma_{h}(0)\right)^{3}$, лемма 2.3 доставляет неравенство Корна (2.8) на $G_{h}^{+}$для $\chi_{h} v$, и поэтому выполняется соотношение

$$
\left|v ; G_{h}^{+}\right|^{2} \leqslant c\left(\left\|v ; g_{h}\right\|^{2}+\left|\chi_{h} v ; G_{h}^{+}\right|^{2}\right) \leqslant C \mathscr{E}\left(u ; G_{h}^{+}\right) .
$$

Осталось обработать жесткое смешение $w(x)=d(x-\mathbf{P}(h)) a$ из (2.33). Как и ранее, оценки (2.36)-(2.38) компонент столбца $a$ позволяют подобрать такую норму $\|\cdot\|$, что

$$
\begin{aligned}
\left\|w ; G_{h}^{+}\right\|^{2} & \leqslant c \operatorname{mes}_{3} G_{h}^{+}\left(\mu_{1}(h)\left(\left|a_{3}\right|^{2}+h^{2}\left|a^{\prime \prime}\right|^{2}\right)+h^{2} \mu_{3}(h)\left(\left|a_{1}\right|^{2}+\left|a_{2}\right|^{2}+\left|a^{\prime \prime}\right|^{2}\right)\right) \\
& \leqslant c \mathscr{E}_{\gamma}\left(u ; G_{h}\right) .
\end{aligned}
$$

Она выглядит аналогично $(2.22)$, но $\mu_{p}(h) h$ и $\mu_{1}(h) h^{-1}$ заменяются на $\mu_{p}(h) h^{2}$ и $\mu_{1}(h)$ (ранее дополнительный множитель $h^{-1}$ компенсировал малую длину $O(h)$ обрубка $g_{h}$ ). Однако такая норма неудовлетворительна (первый раз в статве!) из-за нарушения неравенства $\left\|v ; G_{h}^{+}\right\| \leqslant c\left|v ; G_{h}^{+}\right|$. Для сохранения неравенства приходится заменить в $(2.22)$ весовые множители $\mu_{p}(h) h$ и $\mu_{1}(h) h^{-1}$ на $\mu_{0}(h) h^{2}$ и $\mu_{0}(h)$ соответственно.

Теорема 4.2. Для $u \in \stackrel{\circ}{H^{1}}\left(G_{h} ; \Gamma_{h}\left(-L_{-}\right)\right)^{3}$ справедливо весовое анизотропное неравенство Корна

$$
\gamma(h)\left|u ; G_{h}^{-}\right|^{2}+\left\|u ; G_{h}^{+}\right\|^{2} \leqslant c\left(\gamma(h) \mathscr{E}\left(u ; G_{h}^{-}\right)+\mathscr{E}\left(u ; G_{h}^{+}\right)\right),
$$

в котором $|\cdot| u\|\cdot\|-$ нормы (2.9) $и$

$$
\begin{aligned}
\left\|u ; G_{h}^{+}\right\|=( & \int_{G_{h}^{+}}\left(\sum_{i=1}^{2}\left(\left|\frac{\partial u_{i}}{\partial y_{i}}\right|^{2}+h^{2} \mu_{0}(h)\left(\left|\frac{\partial u_{i}}{\partial z}\right|^{2}+\left|\frac{\partial u_{3}}{\partial y_{i}}\right|^{2}\right)+h^{2} \mu_{0}(h)\left|u_{i}\right|^{2}\right)\right. \\
& \left.\left.+\left|\frac{\partial u_{3}}{\partial z}\right|^{2}+h^{2} \mu_{0}(h)\left(\left|\frac{\partial u_{1}}{\partial y_{2}}\right|^{2}+\left|\frac{\partial u_{2}}{\partial y_{1}}\right|^{2}\right)+\mu_{0}(h)\left|u_{3}\right|^{2}\right)\right)^{1 / 2},
\end{aligned}
$$

a $\mu_{0}(h)=\min \{1, \gamma(h)\}-$ величина $(2.23)$.

В случае $\gamma(h)=1$ норма (4.7) преврашается в (2.9), а оценка (4.6) - в оценку (2.8). Отметим, что при помоши подхода, намеченного в [38], можно установить асимптотически точные неравенства Корна для сочленений двух разнородных стержней.

4.4. Стержень, присоединенный к защемленной пластине. Пусть $\tau$ непустая открытая дуга контура $\partial \omega^{0}$ и $\mathscr{T}_{h}^{0}=\tau \times(-h / 2, h / 2)$. Рассмотрим сочленение (1.3) и поле $v \in \stackrel{\circ}{H}{ }^{1}\left(\Omega(h) ; \mathscr{T}_{h}^{0}\right)^{3}$. Механическая интерпретация включения такова: пластина защемлена вдоль части $\mathscr{T}_{h}^{0}$ ее боковой поверхности, но торцы $\Gamma_{h}^{j 0}$ стержней $G_{h}^{j}$ свободны. Построим нормы $\left\|\cdot ; G_{h}^{j}\right\|_{j}$, обеспечивающие неравенство Корна (1.8) в указанном случае. Для краткости имеем дело только со стержнями, вставленными в пазы и являюшимися не менее жесткими, чем пластина (т.е. $\left.\gamma(h) \geqslant \gamma_{0}>0\right)$. 
Поскольку $v=0$ на $\mathscr{T}_{h}^{0}$, неравенство Корна (2.3) выполняется без условия (2.2) и сохраняется при следуюшем усилении нормы (2.4):

$$
\begin{aligned}
\left|v ; \Omega_{h}^{0}\right|_{0}= & \left(\int _ { \Omega _ { h } ^ { 0 } } \left(\sum_{i=1}^{2}\left(\left|\frac{\partial v_{i}}{\partial y_{i}}\right|^{2}+h^{2} \rho_{h}^{-2}\left(\left|\frac{\partial v_{i}}{\partial z}\right|^{2}+\left|\frac{\partial v_{3}}{\partial y_{i}}\right|^{2}\right)+\rho_{h}^{-2}\left|v_{i}\right|^{2}\right)\right.\right. \\
& \left.\left.+\left|\frac{\partial v_{3}}{\partial z}\right|^{2}+\left|\frac{\partial v_{1}}{\partial y_{2}}\right|^{2}+\left|\frac{\partial v_{2}}{\partial y_{1}}\right|^{2}+h^{2} \rho_{h}^{-4}\left|v_{3}\right|^{2}\right)\right)^{1 / 2}
\end{aligned}
$$

(см. [18; теорема 3.3.3], а также [12], [13]); в $(4.8) \rho_{h}(x)=h+\operatorname{dist}\{y, \tau\}$. Так как $\rho_{h}(x) \leqslant C h$ на $c h$-окрестности $\Theta_{h}^{0}$ множества $\mathscr{T}_{h}^{0}$, имеем

$$
\left\|v ; \Omega_{h}^{0} \cap \Theta_{h}^{0}\right\|^{2} \leqslant c\left|v ; \Omega_{h}^{0}\right|^{2} \leqslant c \mathscr{E}\left(v ; \Omega_{h}^{0}\right) .
$$

Поэтому выкладки (4.4), (4.5) и неравенство (2.8) для поля $v$, умноженного на срезку $\chi_{h}$, показывают, что для стержней, присоединенных в непосредственной близости от зажатой поверхности $\mathscr{T}_{h}^{0}$, в качестве $\|\cdot\|_{j}$ можно взять норму $(2.9)$, в которой весовой множитель $\rho_{h}(x)$ равен $h+\operatorname{dist}\left\{x, g_{h}^{j}\right\}$.

Пусть стержень $G_{h}^{j}$ удален от $\mathscr{T}_{h}^{0}$ и $B_{h}^{j} \subset g_{h}^{j}$ - шар с радиусом $h R_{j}$ и центром $\mathbf{P}^{j}(h)$. Разложим поле ${ }^{3} u \in \stackrel{\circ}{H}^{1}\left(\Omega(h), \mathscr{T}_{h}^{0}\right)^{3}$ на шаре $B_{h}^{j}$ согласно $(2.33)$ и распространим это разложение на весь стержень. Обрашаясь к формулам (2.18), (2.35) и (4.3)-(4.5), заключаем, что

$$
\left|v ; G_{h}^{j}\right|_{j}^{2} \leqslant c \mathscr{E}\left(u ; G_{h}^{j}\right),
$$

причем $|\cdot|_{j}$ - норма $(2.9)$ с указанной вьше весовой функцией $\rho_{h}$. Теперь занимаемся столбцом $a$ из (2.33), удовлетворяющим системе (3.2) с матрицей (3.15) и правой частью (3.4). Поступая так же, как и в предыдущих пунктах, выводим при помощи неравенств $(2.18)$ с $\Xi_{h}^{p}=B_{h}^{j}$ и $(2.39),(2.40)$ оценки

$$
\begin{aligned}
\left|a_{i}\right|^{2} & \leqslant c h^{-3} \int_{B_{h}^{j}}\left(\left|u_{i}\right|^{2}+\left|v_{i}\right|^{2}\right) d x \leqslant c h^{-3}\left(h^{2}|\log h|^{2}+h^{2} \gamma(h)^{-1}\right) \mathscr{E}_{\gamma}(u ; \Omega(h)) \\
& \leqslant c h^{-1} \lambda_{0}(h)^{-1} \mathscr{E}_{\gamma}(u ; \Omega(h)), \\
\left|a_{3}\right|^{2} & \leqslant c h^{-3} \int_{B_{h}^{j}}\left(\left|u_{3}\right|^{2}+\left|v_{3}\right|^{2}\right) d x \leqslant c h^{-3}\left(|\log h|^{2}+h^{2} \gamma(h)^{-1}\right) \mathscr{E}_{\gamma}(u ; \Omega(h)) \\
& \leqslant c h^{-1} \lambda_{-2}(h)^{-1} \mathscr{E}_{\gamma}(u ; \Omega(h)), \\
\left|a^{\prime \prime}\right|^{2} & \leqslant c h^{-5} \int_{B_{h}^{j}}\left(|u-\bar{u}|^{2}+|v|^{2}\right) d x \leqslant c h^{-5}\left(h^{2}|\log h|^{2}+h^{2} \gamma(h)^{-1}\right) \mathscr{E}_{\gamma}(u ; \Omega(h)) \\
& \leqslant c h^{-3} \lambda_{0}(h)^{-1} \mathscr{E}_{\gamma}(u ; \Omega(h)) .
\end{aligned}
$$

Ввиду различия индексов $p$ у множителей $\lambda_{p}(h)$ (см. (1.10)) в мажорантах из $(4.11)$ и $(4.10),(4.12)$ приходится при обработке жесткого смешения $w(x)=d\left(x-\mathbf{P}^{j}(h)\right) a$ выделять следующий случай:

6) ось $\Lambda^{j}$ стерэня $G_{h}^{j}$ лежит в плоскости $\{x: z=0\}$.

\footnotetext{
${ }^{3}$ Новое обозначение вводим для привлечения формул из п. 2.3.
} 
Для таких стержней удается улучшить оценку продольной компоненты $u_{3}^{j}$, которая оказывается не зависящей от элемента $a_{3}$ с наихудшей мажорантой в (4.10), (4.11). Наконец, при совмешении установленных соотношений для $w$ и $v$ наблюдается тот же эффект, что и в предыдушем пункте: весовые множители в окончательной норме определяются обеими возникшими нормами. Опуская рутинные вычисления, формулируем результат.

TEOPEMA 4.3. Пусть $\gamma(h) \geqslant \gamma_{0}>0 u u \in \stackrel{\circ}{H^{1}}\left(\Omega(h) ; \mathscr{T}_{h}^{0}\right)^{3}$. Выполняется неравенство Корна $(1.8)$, в котором норма $\|\cdot\|_{0}$ на пластине задается формулой (4.8) с весовой функиией $\rho_{h}(x)=h+\operatorname{dist}\{y, \tau\}$. При $P^{j} \in \bar{\tau}$ норма $\|\cdot\|_{j}$ на стержне $G_{h}^{j}$ имеет вид (2.9), где $\rho_{h}(x)=h+\operatorname{dist}\left\{x, g_{h}^{j}\right\}$, но при $P^{j} \in \overline{\omega^{0}} \backslash \bar{\tau}$

$$
\begin{aligned}
\left\|u ; G_{h}^{j}\right\|_{0}=( & \gamma(h)^{-1} \int_{G_{h}^{j}}\left(\sum _ { i = 1 } ^ { 2 } \left(\gamma(h)\left|\frac{\partial u_{i}^{j}}{\partial y_{i}^{j}}\right|^{2}+h^{2} \lambda_{1}(h)\left(\left|\frac{\partial u_{i}^{j}}{\partial z^{j}}\right|^{2}+\left|\frac{\partial u_{3}^{j}}{\partial y_{i}^{j}}\right|^{2}\right)\right.\right. \\
& \left.+h \lambda_{1}(h)\left|u_{i}^{j}\right|^{2}\right)+\left|\frac{\partial u_{3}^{j}}{\partial z^{j}}\right|^{2} \\
& \left.\left.+h^{2} \lambda_{1}(h)\left(\left|\frac{\partial u_{1}^{j}}{\partial y_{2}^{j}}\right|^{2}+\left|\frac{\partial u_{2}^{j}}{\partial y_{1}^{j}}\right|^{2}\right)+\lambda_{-1}(h)\left|u_{3}^{j}\right|^{2}\right)\right)^{1 / 2}
\end{aligned}
$$

причем в случае 6) вместо $\lambda_{-1}(h)\left|u_{3}^{j}\right|^{2}$ в (4.13) фигурирует $\lambda_{1}(h)\left|u_{3}^{j}\right|^{2}$.

Подчеркнем, что $\lambda_{-1}(h)=h|\log h|^{-2}$ при введенном требовании $\gamma(h) \geqslant \gamma_{0}$. Кроме того, $\lambda_{1}(h)=\gamma(h)$ для "не очень жестких" стержней (т.е. $\gamma(h) \leqslant \gamma_{1} h^{-1} \times$ $|\log h|^{-2}$; ср. (3.30)), а значит, в случае 6) норма (4.13) не отличается от нормы $(2.9)$, в которой $\rho_{h}=1$.

4.5. Пластина, защемленная по малым множествам. На $\Omega_{h}^{0}$ рассмотрим поле $u \in \stackrel{\circ}{H}^{1}\left(\Omega_{h}^{0} ; \mathscr{T}_{h}^{1} \cup \cdots \cup \mathscr{T}_{h}^{J}\right)^{3}$. Благодаря условию Дирихле $u=0$ на $\mathscr{T}_{h}^{j} \subset \partial \Omega_{h}^{0}$ вьполняется аналогичное (2.18) неравенство Корна

$$
\left\|u ; \Theta_{h}^{j} \cap \Omega_{h}^{0}\right\|^{2} \leqslant c \mathscr{E}\left(u ; \Theta_{h}^{j} \cap \Omega_{h}^{0}\right),
$$

где $\Theta_{h}^{j}=\left\{x:\left|y-P^{j}\right| \leqslant \mathscr{R}_{j} h\right\}-$ тонкий цилиндр, содержащий множество $\mathscr{T}_{h}^{j}$. Умножим $u$ на срезаюшую функцию $X_{h} \in C^{\infty}\left(\overline{\Omega_{h}^{0}}\right)$, равную единице вне $\Theta_{h}^{1} \cup$ $\cdots \cup \Theta_{h}^{J}$ и нулю при $\left|y-P^{j}\right| \leqslant \mathscr{R}_{j} h / 2$. Как и в (4.4),

$$
\mathscr{E}\left(X_{h} u ; \Omega_{h}^{0}\right) \leqslant c\left(\mathscr{E}\left(u ; \Omega_{h}^{0}\right)+h^{-2} \sum_{j=1}^{J}\left\|u ; L_{2}\left(\Theta_{h}^{j} \cap \Omega_{h}^{0}\right)\right\|^{2}\right) \leqslant c \mathscr{E}\left(u ; \Omega_{h}^{0}\right) .
$$

Сохраним обозначение $u$ для произведения $X_{h} u$ и выберем радиус $\mathscr{R}_{j}$ так, чтобы $u=0$ на шарах $B_{h}^{j}$ - областях интегрирования в $(3.4),(3.7),(3.13)$ и пр. Другими словами, из формул для правых частей $F^{j}, \mathscr{F}^{j}, \mathbf{F}^{1}$ и т.п. исчезает слагаемое $u(y, z)$, обслуживаемое леммами 2.4 и 2.5 . Приспособить оценки из $\S 3$ к возникшей ситуации нетрудно: достаточно формально положить $\gamma(h)=+\infty$. При этом согласно определениям (2.23) и (1.10) $\mu_{q}(h)=h^{-q}$ и $\lambda_{q}(h)=h^{-q}|\log h|^{-2}$ во всех нормах, возникших в $\S 2$ и $\S 3$. Ограничимся пересказом лишш одного результата, относяшегося к норме (3.49) и следуюшему геометрическому требованию:

7) среди точек $P^{1}, \ldots, P^{J}$ найдутся три, совпадающие с вершинами невирожденного треугольника. 
Отметим, что из упомянутых вершин можно вьпустить по два фиктивных бесконечно жестких стержня и тем самым соблюсти оба условия 4) и 5).

ТЕОРемA 4.4. Пусть $J \geqslant 3$ и справедливо условие 7). Тогда для поля $u \in H^{1}\left(\Omega_{h}^{0} ; \mathscr{T}_{h}^{1} \cup \cdots \cup \mathscr{T}_{h}^{J}\right)^{3}$ справедливо неравенство Корна

$$
\begin{gathered}
\int_{\Omega_{h}^{0}}\left(\sum_{i=1}^{2}\left(\left|\frac{\partial u_{i}}{\partial y_{i}}\right|^{2}+h^{2}|\log h|^{2}\left(\left|\frac{\partial u_{i}}{\partial z}\right|^{2}+\left|\frac{\partial u_{3}}{\partial y_{i}}\right|^{2}\right)+|\log h|^{2}\left|u_{i}\right|^{2}\right)+\left|\frac{\partial u_{3}}{\partial z}\right|^{2}\right. \\
\left.+|\log h|^{2}\left(\left|\frac{\partial u_{1}}{\partial y_{2}}\right|^{2}+\left|\frac{\partial u_{2}}{\partial y_{1}}\right|^{2}\right)+h^{2}|\log h|^{2}\left|u_{3}\right|^{2}\right) d x \leqslant c \mathscr{E}\left(u ; \Omega_{h}^{0}\right)
\end{gathered}
$$

в котором постоянная с не зависит от и $и h \in\left(0, h_{0}\right]$.

Обрашаем внимание на то, что по сравнению с обычной нормой (2.4) в норме, квадрат которой стоит в левой части (4.14), возникли лишь дополнительные логарифмические множители. Другими словами, при игнорировании в норме (4.8) весовой функции $\rho_{h}$, проявляющейся лишш вблизи $\mathscr{T}_{h}^{0}$, защемление пластины по трем множествам с диаметрами $O(h)$, расположенными не вдоль одной прямой, “почти равносильно" закреплению пластины по всей боковой поверхности.

\section{Список литературы}

1. Физики продолжают путить. М.: Мир, 1968.

2. Friedrichs K. O. On the boundary value problems of the theory of elasticity and Korn's inequality // Ann. of Math. (2). 1947. V. 48. P. 441-471.

3. Nečas J. Les méthodes in théorie des équations elliptiques. Paris-Prague: Masson-Academia, 1967.

4. Дюво Г., Лионс Ж.-Л. Неравенства в механике и физике. М.: Наука, 1980.

5. Кондратьев B. А., Олейник O.A. Краевые задачи для системы теории упругости в неограниченных областях. Неравенство Корна // УМН. 1988. Т. 43. № 5. С. 55-98.

6. Cioranescu D., Oleinik O. A., Tronel G. On Korn's inequalities for frame type structures and junctions // C. R. Acad. Sci. Paris Sér. I Math. 1989. V. 309. P. 591-596.

7. Kozlov V.A., Maz'ya V. G., Movchan A.B. Asymptotic representation of elastic fields in a multi-structure // Asymptot. Anal. 1995. V. 11. P. 343-415.

8. Kozlov V.A., Maz'ya V.G., Movchan A. B. Asymptotic analysis of fields in multi-structures. Oxford: Clarendon Press, 1999.

9. Назаров С. А. Неравенства типа неравенств Корна для упругих мултиструктур // УМH. 1995. Т. 50. №6. С. 197-198.

10. Nazarov S. A. Korn's inequalities for junctions of spatial bodies and thin rods // Math. Methods Appl. Sci. 1997. V. 20. №3. P. 219-243.

11. Назаров C.A. Асимптотика решений задачи теории упругости для трехмерного тела с тонкими отростками // Докл. РАН. 1997. Т. 352. № 4. С. 458-461.

12. Шойхет Б. А. Об асимптотически точных уравнениях тонких плит сложной структуры // Прикл. матем. и мех. 1973. Т. 37. № 5. С. 913-924.

13. Назаров С.А. Неравенства Корна, асимптотически точные для тонких областей // Вестн. СПбГУ. Сер. 1. 1992. № 2(8). С. 19-24.

14. Назаров С. А. Обоснование асимптотической теории тонких стержней. Интегральные и поточечные оценки // Проблемы математического анализа. Т. 17. СПб.: Изд-во СПбГУ, 1997. C. $101-152$.

15. Назаров С. А. Асимптотический анализ произвольно анизотропной пластины переменной толщины (пологой оболочки) // Матем. сб. 2000. Т. 191. № 7. С. 129-159.

16. Назаров С.А., Слуикий А.C. Одномерные уравнения деформации тонких слабоискривленных стержней. Асимптотический анализ и обоснование // Изв. РАН. Сер. матем. 2000. T. 64. №3. C. 97-131. 
17. Акимова E. A., Назаров C. А., Чечкин Г. А. Весовое неравенство Корна: процедура "тетрис", обслуживающая произвольную периодическую пластину // Докл. РАН. 2001. T. 380. № 4. C. 439-442.

18. Назаров $C$. A. Асимптотическая теория тонких пластин и стержней. Понижение размерности и интегральнте оценки. Новосибирск: Научная книга, 2002.

19. Caillerie $D$. The effect of a thin inclusion of high rigidity in an elastic body // Math. Methods Appl. Sci. 1980. V. 2. №3. P. 251-270.

20. Ciarlet P. G. Plates and junctions in elastic multi-structures: an asymptotic analysis. Paris: Masson, 1988.

21. Ciarlet P. G., Le Dret H., Nzengwa R. Modelisation de la jonction entre un corps elastique tridimensionnel et une plaque // C. R. Acad. Sci. Paris Sér. I Math. 1987. V. 305. P. 55-58.

22. Kozlov V.A., Maz'ya V. G., Movchan A.B. Asymptotic analysis of a mixed boundary value problem in a multi-structure // Asymptot. Anal. 1994. V. 8. P. 105-143.

23. Cioranescu D., Oleinik O. A., Tronel G. Korn's inequalities for frame type structures and junctions with sharp estimates for the constants // Asymptot. Anal. 1994. V. 8. P. 1-14.

24. Назаров С.А. Соединения сингулярно вырождающихся областей различных предельных размерностей. 1, 2 // Труды сем. им. И.Г. Петровского. Т. 18. М.: Изд-во МГУ, 1995. C. $3-78$; T. 20. 1997. C. $155-195$.

25. Nazarov S. A. Junction problem of bee-on-ceiling type in the theory of anisotropic elasticity // C.R. Acad. Sci. Paris Sér. I Math. 1995. V. 320. № 11. P. 1419-1424.

26. Аргатов И. И., Назаров С. А. Асимптотический анализ на соединениях областей различных предельных размерностей. Тело, пронзенное тонким стержнем // Изв. РАН. Сер. матем. 1996. Т. 60. №1. С. 3-36.

27. Аргатов И.И., Назаров С.А. Асимптотический анализ на соединениях областей различных предельных размерностей. Упругое тело, пронзенное тонкими стержнями // Проблемы математического анализа. Т. 20. Новосибирск: Научная книга, 2000. С. 3-55.

28. Мельник T. A., Назаров С. А. Асимптотический анализ задачи Неймана на соединении тела с тонкими тяжельми стержнями // Алгебра и анализ. 2000. Т. 12. № 2. С. 188-238.

29. Бахвалов Н. С., Панасенко Г. П. Осреднение процессов в периодических средах. Математические задачи механики композиционных материалов. М.: Наука, 1984.

30. Cioranescu D., Saint Jean Paulin J. Structures très minces en elasticité linéarisée: tours et grillages // C.R. Acad. Sci. Paris Sér. I Math. 1989. V. 308. P. 41-46.

31. Панасенко Г. П. Асимптотические решения системы теории упругости для стержневых и каркасных структур // Матем. сб. 1992. Т. 183. № 1. С. 89-113.

32. Panassenko G. P. Asymptotic analysis of bar systems. 1, 2 // Russian J. Math. Phys. 1994. V. 2. № 3. P. $325-352$; 1996. V. 4. № 1. P. 87-116.

33. Cioranescu D., Jean Paulin J.S. Homogenization of reticulated structures. Berlin: Springer-Verlag, 1999. (Appl. Math. Sci. V. 136.)

34. Zhikov V. V. Homogenization of elasticity problems on singular structures // Preprint № 1. Vladimir: State Pedagogical Univ., 2000.

35. Le Dret H. Modeling of the junction between two rods // J. Math. Pures Appl. (9). 1989. V. 68. P. 365-397.

36. Le Dret $H$. Problemes variationnels dans les multi-domains. Modélisation des jonctions et applications. Paris: Masson, 1991.

37. Назаров C.A., Слуцкий A.C. Асимптотика частот собственных колебаний упругих балок, соединенных в форме буквы П // Докл. РАН. 2001. Т. 380. №1. С. 23-26.

38. Назаров C. А., Слуцкий A. C. Произвольные плоские системы анизотропных балок // Труды МИАН. 2002. Т. 236. С. 234-261.

39. Назаров С. А., Пламеневский Б. А. Асимптотика спектра задачи Неймана в сингулярно вырождающейся тонкой области. 1 // Алгебра и анализ. 1990. Т. 2. № 2. С. 85-111.

Институт проблем машиноведения РАН,

г. Санкт-Петербург

E-mail: serna@snark.ipme.ru
Поступила в редакцию 30.10 .2002 и 01.12 .2003 